\title{
Local Variations on a Loose Theme: Modal Logic and Decidability
}

\author{
Maarten Marx and Yde Venema
}

\subsection{Introduction}

This chapter is about decidability and complexity issues in modal logic; more specifically, we confine ourselves to satisfiability (and the complementary validity) problems. The satisfiability problem is the following: for a fixed class of models, to determine whether a given formula $\varphi$ is satisfiable in some model of that class (a more precise definition will follow). The general picture is that modal logic behaves quite well in this respect. In fact, many authors follow Vardi [58] in calling modal logic robustly decidable on the ground that most of the nice computational properties of modal logic are preserved if one considers extensions or variants of the basic system. The main aim of this chapter is to refine and analyze this picture.

To start with, we should clarify what we are talking about when using the term "modal logic". Traditionally, propositional modal logic would be described as an extension of propositional logic with operators $\square$ and $\diamond$ for talking about the necessity and possibility of a formula being true. However, nowadays the term "modal logic" is used for a plethora of formalisms, with applications in various disciplines ranging from linguistics to economics, see $[11,17,39,56]$ for a sample of applications in computer science.

And while (propositional) modal logics will usually still be an extension of classical propositional logic with a number of modal operators, the intended meanings of these operators differ enormously. For instance, the formula $\square_{a} \varphi$ could mean "player $a$ knows that $\varphi$ is the case" in a formalization of game theory, or "after the execution of program $a, \varphi$ will be the case" in a formal language for program verification. Fortunately, on a technical level, all these formalisms still have a lot in common. That is why this chapter first introduces the notion of a modal system as a triple consisting of a (propositional) modal language, a class of models and a truth function. This definition covers most of the systems that appear in the literature under the name "modal logic"; in particular, the familiar system of basic modal logic, to be discussed in section 7.3 . 
Now that we know what modal logic is, can we say what makes it so robustly decidable? If we confine ourselves to basic modal logic, the answer seems to be affirmative. As we shall see further on, the fact that the truth of basic modal formulas is invariant under bisimulations ensures that basic modal logic has the tree model property. That is, every satisfiable modal formula is satisfiable in a special, "loose", model based on a tree. This makes it much easier to check whether a given modal formula is satisfiable: one only needs to worry about these loose models. And since the bisimulation invariance property transfers to many extensions and variants of the basic modal system, so does the decidability of the satisfiability problem.

This analysis, due to Vardi [58], in terms of a looseness principle, will form the main theme of our chapter. However, it can only form part of the story. For instance, suppose that we are interested not just in decidability, but also in the computational complexity of the satisfiability problem. Not all loose modal systems are in the same complexity class, so there must be principles besides looseness that determine the computational behavior of a modal system. Or, what if we happen to be working with a modal system that does not allow trees as models? Will this necessarily make the logic undecidable? Answers to such questions cannot be precise and general at the same time - note that the problem of whether a given modal axiom determines a decidable modal logic ("logic" here in the technical sense; see below), is itself undecidable! Nevertheless, we believe that it is possible to provide some rough guidelines, and we shall discuss some of these in this chapter; in particular, at the end of section 7.3, we discuss two locality principles.

Thus, it is our aim to act as the reader's travel guide in the landscape of modal logics by pointing out some interesting decidability and complexity theoretic phenomena and by suggesting an interpretation of these phenomena as local variations on a loose theme. On the trip we shall introduce some important modal systems and proof methods - but we have not aimed for a complete or systematic overview in this respect. (For instance, we shall not employ any automata-theoretic methods.) No previous exposure of the reader to modal logic is assumed. Finally, we do not usually provide credits or give references in running text; these are supplied in the "Notes" paragraphs that finish each section.

Overview of chapter In the next section we explain our interpretation of the terms "modal logic" and "modal systems", and we define the notion of bisimulation. Section 7.3 discusses basic modal logic, giving a detailed proof of the decidability of its satisfiability problem; analyzing this proof, we introduce the notions of looseness and locality. In the section after that, we use a number of examples to show what happens if we play around a bit with these principles. Section 7.5 is devoted to a more fine-grained, complexity-theoretic study of the modal satisfiability problem. In the last section, we show how one can use the principles discussed in the earlier parts of the chapter to find large fragments of ordinary first-order logic that have a decidable satisfiability problem. 


\subsection{Modal Systems and Bisimulations}

In this section, we discuss our interpretation of the term "modal logic" by defining and explaining the notion of a modal system. We also introduce the fundamental notion of similarity between two modal models, namely that of a bisimulation. The link between modal logic and bisimulation is that modal formulas cannot distinguish bisimilar points.

\section{Modal systems}

A modal system consists of a modal language $\mathcal{L}$, a class of models $\mathrm{K}$, and a function $\Vdash$ interpreting formulas of the language in the models. As models, we shall consider only relational structures, that is, structures consisting of a nonempty domain or universe together with a number of relations on it. By the size of a model $\mathcal{M}$, we always denote the size of its domain. The elements of the universe of a model will be called states, points, or worlds. In the modal part of this chapter we shall confine ourselves to models that have a number of binary relations (usually just one, which we denote by $R$ ) and a countable number of unary relations $P_{0}, P_{1}, \ldots$ See Figure 7.1 for a graphical presentation of these models.
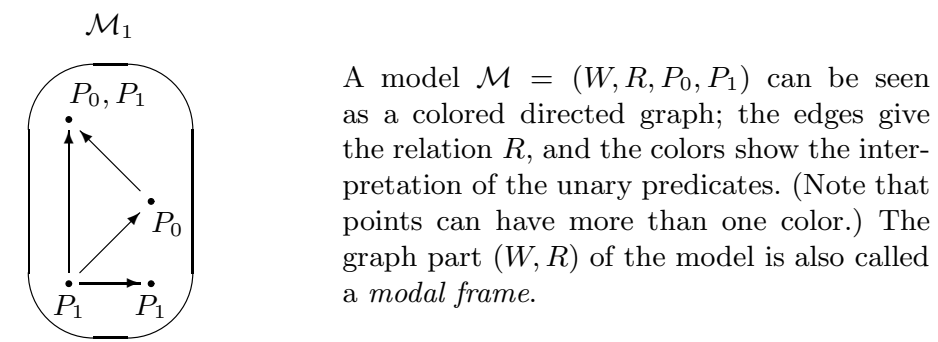

Fig. 7.1. Graphical representation of a model.

A modal language is a simple yet expressive language for talking about such relational structures. In this chapter we consider only propositional modal languages; these can be described as extensions of the classical propositional language with a collection of modal connectives such as the unary modal operator $\diamond$. Like the boolean connectives, the modal operators do not bind variables. The size of a formula $\varphi$ (notation $|\varphi|$ ) in a modal language $\mathcal{L}(\Phi)$ (i.e., with propositional variables from a set $\Phi$ ) is its length over the alphabet $\Phi \cup\left\{\neg, \wedge,(,), \nabla_{i}\right\}_{i \in I}$, where $\left\{\nabla_{i} \mid i \in I\right\}$ denotes the set of modal connectives of $\mathcal{L}$.

Finally, $\Vdash$ is a function which takes a model $\mathcal{M}$ and a formula $\varphi$ and returns a subset of the domain of $\mathcal{M}$ that we shall think of as the meaning 
of $\varphi$ in the model. The standard terminology for stating that $s \in \Vdash(\mathcal{M}, \varphi)$ is that $\varphi$ is true or holds at $s$ in $\mathcal{M}$, and the standard notation is

$$
\mathcal{M}, s \Vdash \varphi .
$$

The meaning of the propositional variable $p_{i}$ is the corresponding set $P_{i}$, and thus

$$
\mathcal{M}, s \Vdash p_{i} \leftrightarrow P_{i} s .
$$

For the Boolean connectives we have the standard interpretation in mind, which requires that $\neg \varphi$ is true at a state iff $\varphi$ is false (i.e., not true) at it, and that $\varphi \wedge \psi$ holds precisely at those states where both $\varphi$ and $\psi$ hold.

The conditions on modal systems mentioned so far still allow for an enormous freedom in defining the semantics of the modal connectives. But even in this very wide and general setting we can introduce the notions of satisfiability and validity associated with such a triple: we call a formula valid if it is true at every state of every model of the system, and satisfiable if it is true at some state of some model of the system. The validity problem of a modal system is the problem of deciding whether a given formula of the language is valid or not; the satisfiability problem is defined analogously. Given our constraints on the interpretation of Boolean negation, it is obvious that a formula $\xi$ is valid in a class $\mathrm{K}$ of models if and only if its negation $\neg \xi$ is not satisfiable in any model in K. Hence, for any class of models K, there are constant-time reductions between the satisfiability problem and the complement of the validity problem. We shall use this fact in what follows without explicit mention; also, we shall use the term "complexity of a modal system" when referring to its satisfiability problem.

Each of the three ingredients of a modal system - the language $\mathcal{L}$, the class $\mathrm{K}$ of models, and the interpretation function $\Vdash$ - influences the complexity of the satisfiability problem. We shall see that many important and interesting modal systems have a decidable satisfiability problem, but the above definition of a modal system is also wide enough to allow for systems whose satisfiability problem is highly undecidable. Our aim in this chapter is to provide some rough guidelines for determining the complexity of a modal system. For the sake of a simple exposition, we first restrict our attention to a simple yet interesting type of models: those of the signature with one binary relation $R$ and a number of unary relations. Even with this signature fixed, we still have an enormous freedom in defining the modal language $\mathcal{L}$ and the meaning of the modal connectives given by the function $\Vdash$. What, then, the reader will ask, is particularly modal about a system? We shall now state a further restriction on modal systems which is very characteristic of modal logic. It concerns the discriminatory power of modal languages. 


\section{Bisimulation}

An informative way to identify a language is by saying which differences between models it is blind to. In the case of modal logic, the fundamental concept of equivalence between structures involves the notion of bisimulation.

Definition 7.2.1. Given two models $\mathcal{M}=\left(W, R, P_{i}\right)_{i \in I}$ and $\mathcal{M}^{\prime}=$ $\left(W^{\prime}, R^{\prime}, P_{i}^{\prime}\right)_{i \in I}$, a nonempty relation $Z \subseteq W \times W^{\prime}$ is a bisimulation between $\mathcal{M}$ and $\mathcal{M}^{\prime}$ if the following three conditions hold, for all states $s \in W$ and $s^{\prime} \in W^{\prime}$ that are linked by $Z$ :

(base) for all $i: P_{i} s$ iff $P_{i}^{\prime} s^{\prime}$;

(forth) for all $t \in W$ such that Rst, there is a $t^{\prime} \in W^{\prime}$ with $R^{\prime} s^{\prime} t^{\prime}$ and $t Z t^{\prime}$;

(back) for all $t^{\prime} \in W^{\prime}$ such that $R^{\prime} s^{\prime} t^{\prime}$, there is a $t \in W$ with Rst and $t Z t^{\prime}$.

If there is some bisimulation $Z$ linking $s$ and $s^{\prime}$, then we say that $s$ and $s^{\prime}$ are

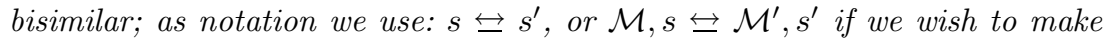
the models explicit.

Figure 7.2 contains two simple examples of bisimulating models (the models bisimulate horizontally) in a language with only one unary relation $P$. Figure 7.3 shows two models which do not bisimulate at the roots. All states in both models satisfy the same unary relations; $\mathcal{M}^{\prime}$ has all of the finite branches that $\mathcal{M}$ has, but in addition it contains an infinite branch.
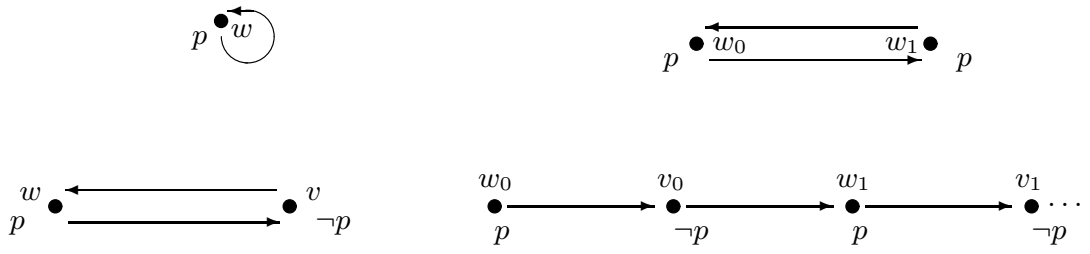

Fig. 7.2. Two examples of bisimulating models

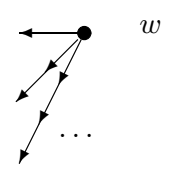

$\mathcal{M}$

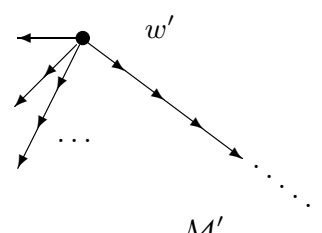

$\mathcal{M}^{\prime}$

Fig. 7.3. $\mathcal{M}, w$ and $\mathcal{M}^{\prime}, w^{\prime}$ are not bisimilar 
We can now make the crucial restriction on $\Vdash$ precise: we want the truth of modal formulas to be invariant under bisimulations. That is, our basic interest is in a truth definition for the modal connectives that makes the semantics satisfy the following constraint:

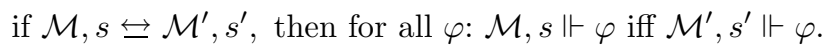

We shall call any system in which $\Vdash$ meets this constraint a modal system in the narrow sense.

But why would we be interested in a language that cannot see the difference between bisimilar states (if not for technical reasons)? Apart from the original logical considerations that we are about to describe, an important reason stems from theoretical computer science. Here, or more specifically in the field of process theory, one models processes as labeled transition systems; these are relational structures like the one we describe here, though usually with a collection of binary relations instead of just one. The idea here is that a state $s$ in a model $\mathcal{M}$ represents some state of the process: the predicates $P_{i}$ correspond to various direct observations that we can make about states, and the relations $R_{j}$ correspond to the various transition steps that the process may take. A pair $(s, t)$ belonging to the relation $R_{j}$ indicates that at $s$ the process can take an $R_{j}$-step, thus reaching the state $t$, where new direct observations can be made, or new steps can be taken. Now, in this context, states that are bisimilar cannot be distinguished from a process-theoretic point of view and thus represent the same state. Thus, bisimulation serves as one of the most fundamental notions of identity between process states. This explains why languages designed for expressing properties of processes should indeed be blind to the distinction between bisimilar states.

Let us turn to some concrete examples. We first give some examples of operators which meet this requirement:

$$
\begin{gathered}
\mathcal{M}, s \Vdash \diamond \varphi \text { if } \mathcal{M}, t \Vdash \varphi \text { for some } t \text { such that } R s t ; \\
\mathcal{M}, s \Vdash\langle *\rangle \varphi \text { if there is some path } s=s_{0} R s_{1} R s_{2} \ldots R s_{n}=t \text { through } \mathcal{M} \\
\text { such that } \mathcal{M}, t \Vdash \varphi \text { (including the empty path); } \\
\mathcal{M}, s \Vdash \vec{\infty} \varphi \text { if there is some path } s=s_{0} R s_{1} R s_{2} \ldots \text { through } \mathcal{M} \\
\text { such that } \mathcal{M}, s_{i} \Vdash \varphi \text { for infinitely many } i .
\end{gathered}
$$

We shall show later on that indeed, $\diamond$ does not break the bisimulation invariance (see (7.2) below; the proofs for the other operators are left to the reader).

The operators $\langle\neq\rangle, \mathrm{E}, \mathrm{P}$, and $U$ defined below are not invariant under bisimulations. Figure 7.4 shows two models $\mathcal{M}_{1}, \mathcal{M}_{2}$, both of signature $R, P$, and worlds $s_{1}, s_{2}$, which bisimulate. The valuation of $P$ is indicated in the models. Since both $s_{1}$ and $s_{2}$ have no successors, they bisimulate because they are both not in $P$. It is easy to see that $\langle\neq\rangle p, \mathrm{E} p$ and $\mathrm{P} p$ are all true at $s_{1}$ and all false at $s_{2}$. A counterexample to the bisimulation invariance of the binary connective $U$ is provided just above Figure 7.7 on page 399 . The semantics of the operators is defined as follows: 

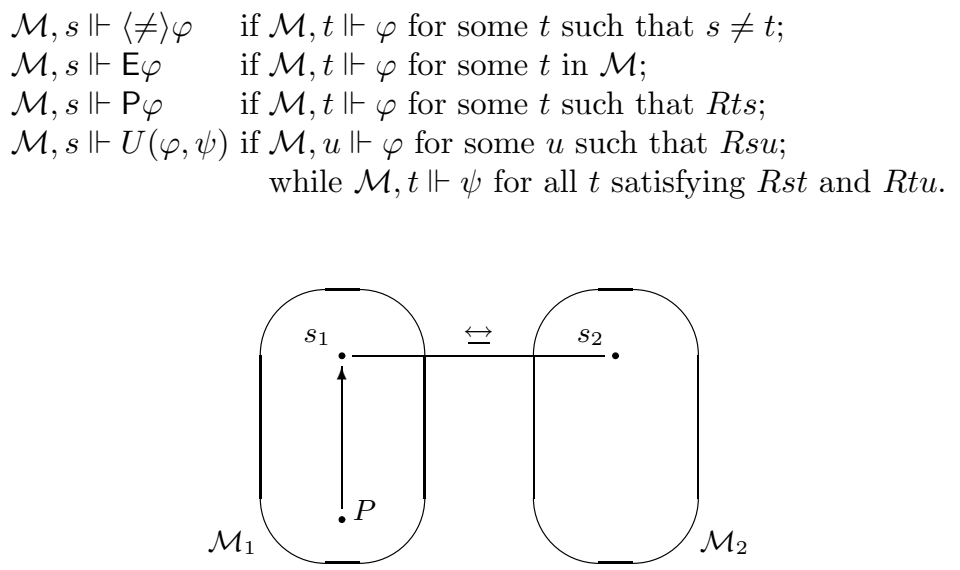

Fig. 7.4. The modal operators $\langle\neq\rangle, \mathrm{E}$ and $\mathrm{P}$ are not invariant under bisimulations.

It seems that $\diamond$ is the simplest nontrivial operator that satisfies the condition of bisimulation invariance. The modal system in which we take $\diamond$ as the only modal operator, in which we allow every relational structure (of the appropriate signature) as a model, and in which $\Vdash$ has the standard definition, is called the basic modal logic. It is discussed in detail in section 7.3.

\section{Some comments}

Before turning to the discussion of the basic modal system, a few comments are in place.

First, it was not our intention to give very rigid definitions. For instance, the question of whether the universal diamond $E$ constitutes a modal system in the narrow sense is really dependent on the perspective that one takes. Earlier on we said that $E$ is not bisimulation-invariant, but what if we take as our class of models precisely those in which $R$ is the universal relation on the model (that is, $\forall x y R x y$ )? In this case the truth definition for $\mathrm{E}$ does satisfy the standard, "bisimulation-invariant" clause for the diamond E; the only thing is that now, our class of models is not closed under taking bisimilar models...

Second, in our introductory discussion we avoided the word "logic". In principle, we prefer to use this word in the technical sense only, referring to a set of formulas that satisfies certain closure properties. For instance, a normal modal logic should be closed under the familiar law of Modus Ponens, and under the rule of Necessitation; the latter means that $\square \varphi$ belongs to the logic whenever $\varphi$ does. We can associate such a logic with many modal systems; in particular, when the system's class of models is defined through some property of the binary relation(s) only, the collection of valid formulas 
will form a logic in the technical sense. Thus the validity problem can often be identified with a membership problem, namely that of a formula in a logic. In a number of cases, we shall forget our principles and follow custom in referring to this associated logic instead of to the modal system.

Finally, the term "modal system in the narrow sense" should not be taken too literally; by playing around with the class $\mathrm{K}$ of models, the reader will easily see that our definition covers a wide range of modal logics. In fact, if we allow languages with more than one modality, then even various versions of first-order logic itself, such as the finite-variable fragments, can be seen as modal systems in the narrow sense!

\subsection{Basic Modal Logic}

In this section, we shall introduce the basic system of modal logic and discuss its close connection to the notion of bisimulation. We shall provide a fairly detailed proof of the decidability of the satisfiability problem for basic modal logic and analyze this result in terms of looseness and locality.

Definition 7.3.1. Given a set $\Phi$ of propositional variables, the collection $\mathcal{L}_{\diamond}(\Phi)$ of basic modal formulas in $\Phi$ is given by the following rule:

$$
\varphi::=p|\neg \varphi| \varphi \wedge \psi \mid \diamond \varphi
$$

where $p$ ranges over elements of $\Phi$. This means that $\varphi$ is either an atomic formula consisting of a proposition letter in $\Phi$, or a more complex formula obtained from simpler ones by applying one of the connectives $\neg, \wedge$, or $\diamond$. We shall use the standard Boolean abbreviations and also the modal "box" operator $\square$, where $\square \varphi$ abbreviates $\neg \diamond \neg \varphi$.

If the collection $\Phi$ of proposition letters is either irrelevant or clear from context, we shall frequently omit it, writing $\mathcal{L}_{\diamond}$ instead of $\mathcal{L}_{\diamond}(\Phi)$. In order to interpret the formulas of this language in a model of the kind discussed above, we represent such a model as a triple $\mathcal{M}=(W, R, V)$ where $W$ is a nonempty set of states, $R$ is the binary relation of the model, and $V: \Phi \rightarrow \mathcal{P}(W)$ is a valuation mapping proposition letters to subsets of $W$. Let $\mathrm{M}$ denote the class of all such models.

Definition 7.3.2. Given a model $\mathcal{M}=(W, R, V)$, a state $s \in W$, and a formula $\varphi$, we define the notion of $\varphi$ being true at $s$, denoted by $\mathcal{M}, s \Vdash \varphi$, recursively as follows:

$$
\begin{array}{ll}
\mathcal{M}, s \Vdash p & \text { if } s \in V(p) ; \\
\mathcal{M}, s \Vdash \neg \varphi \quad \text { if } \operatorname{not} \mathcal{M}, s \Vdash \varphi ; \\
\mathcal{M}, s \Vdash \varphi \wedge \psi & \text { if } \mathcal{M}, s \Vdash \varphi \text { and } \mathcal{M}, s \Vdash \psi ; \\
\mathcal{M}, s \Vdash \diamond \varphi \quad \text { if } \mathcal{M}, t \Vdash \varphi \text { for some } t \text { such that Rst. }
\end{array}
$$


If $\varphi$ is true at every state of the model we say that $\varphi$ holds throughout $\mathcal{M}$, denoted by $\mathcal{M} \Vdash \varphi$; if $\varphi$ holds at some state in $\mathcal{M}$, we say that $\varphi$ is satisfiable in $\mathcal{M}$.

The language of the basic modal system is $\mathcal{L}_{\diamond}$, its class of models is $\mathrm{M}$, and $\Vdash$ is as in the first part of this definition. Depending on the context, we let $\mathbf{K}$ denote either the basic modal system itself or its logic, that is, the set of valid formulas in this system.

As the reader can easily verify, it holds that

$$
\mathcal{M}, s \Vdash \square \varphi \text { if } \mathcal{M}, t \Vdash \varphi \text { for all } t \text { such that Rst. }
$$

The first thing we should check is whether we have met our design criterion (7.1) with this definition of $\Vdash$. Suppose that $\mathcal{M}$ and $\mathcal{M}^{\prime}$ are two modal models, and that $Z$ is a bisimulation between $\mathcal{M}$ and $\mathcal{M}^{\prime}$. We shall prove by a formula induction that every basic modal formula $\varphi$ satisfies the following:

for all $s \in W$ and $s^{\prime} \in W^{\prime}: s Z s^{\prime}$ implies that $\mathcal{M}, s \Vdash \varphi$ iff $\mathcal{M}^{\prime}, s^{\prime} \Vdash \varphi$.

We leave the base step and the boolean cases of the inductive step as exercises for the reader, and concentrate on the modal case of the inductive step. Suppose that $\varphi$ is of the form $\diamond \psi$, and assume that $Z$ links the state $s$ in $\mathcal{M}$ to $s^{\prime}$ in $\mathcal{M}^{\prime}$. For reasons of symmetry, it suffices to show that $\mathcal{M}, s \Vdash \varphi$ only if $\mathcal{M}^{\prime}, s^{\prime} \Vdash \varphi$.

Suppose that $\mathcal{M}, s \Vdash \diamond \psi$. By the truth definition, it follows that there is a state $t$ in $W$ such that $R s t$ and $\mathcal{M}, t \Vdash \psi$. From the fact that $s$ and $s^{\prime}$ are linked by the bisimulation $Z$, we may infer that there is some $R$-successor $t^{\prime}$ of $s^{\prime}$ such that $s^{\prime} Z t^{\prime}$. The inductive hypothesis gives us that $\mathcal{M}^{\prime}, t^{\prime} \Vdash \psi$; but we may then conclude from $R^{\prime} s^{\prime} t^{\prime}$ that $\mathcal{M}^{\prime}, s^{\prime} \Vdash \diamond \psi$, which is precisely what we were after. This proves (7.2) and shows that basic modal logic indeed constitutes a bisimulation-invariant system.

\section{Invariance under bisimulation}

So our definition fulfills our design criterion, but how powerful is this modal language precisely? In other words, how many of the bisimulation-invariant properties can we express in this language? It should be obvious from the truth definition of basic modal logic that we can consider $\mathcal{L}_{\diamond}$ as a fragment of firstorder logic. In fact, we have a kind of functional completeness result as long as we consider first-order properties: every first-order definable bisimulationinvariant property is definable by a modal formula. In other words, when it comes to expressing bisimulation-invariant properties, modal logic is just as strong as first-order logic. In order to state this result formally we need a translation from modal to first-order formulas. 
Definition 7.3.3. Assume that we have an enumeration $x=x_{0}, x_{1}, \ldots$ of first-order variables. Consider the following translation of $\mathcal{L}_{\diamond}$-formulas to first-order formulas:

$$
\begin{aligned}
S T_{x_{i}}(p) & =P x_{i} \\
S T_{x_{i}}(\neg \varphi) & =\neg S T_{x_{i}}(\varphi) \\
S T_{x_{i}}(\varphi \wedge \psi) & =S T_{x_{i}}(\varphi) \wedge S T_{x_{i}}(\psi) \\
S T_{x_{i}}(\diamond \psi) & =\exists x_{i+1}\left(R x_{i} x_{i+1} \wedge S T_{x_{i+1}}(\varphi)\right) .
\end{aligned}
$$

When we speak of "the" standard translation of a modal formula $\varphi$, we are usually referring to the formula $S T_{x_{0}}(\varphi)$.

We can now see modal logic as a fragment of first-order logic because every modal formula is equivalent to its standard translation. Formally (but blurring the distinction between a modal and a first-order model a little), we can prove that for every modal formula $\varphi$, for every model $\mathcal{M}$, and for every state $s$ in $\mathcal{M}$ we have the following equivalence:

$$
\mathcal{M}, s \Vdash \varphi \leftrightarrow \mathcal{M}=S T_{x_{0}}(\varphi)\left[x_{0} \mapsto s\right] .
$$

Here $\left[x_{0} \mapsto s\right]$ denotes any assignment which sends $x_{0}$ to $s$. The simple proof of $(7.3)$ is left to the reader.

Observation 1. The map in Definition 7.3.3 has no upper bound on the number of variables used in the first-order translation of a modal formula. However, one could be very parsimonious and define the formulas $S T_{x}(\varphi)$ and $S T_{y}(\varphi)$ through a mutual recursion, of which the interesting clauses run as follows:

$$
\begin{aligned}
& S T_{x}(\diamond \psi)=\exists y\left(R x y \wedge S T_{y}(\varphi)\right) \\
& S T_{y}(\diamond \psi)=\exists x\left(R y x \wedge S T_{x}(\varphi)\right) .
\end{aligned}
$$

This shows that, in fact, the translation of modal logic to first-order logic can be carried out within the two variable fragment of first-order logic. We shall come back to this observation later on.

Now we are ready to state the celebrated Characterization Theorem for modal logic.

Theorem 7.3.4. Let $\varphi(x)$ be a first-order formula in the signature consisting of a binary $R$ and a set $\left\{P_{i} \mid i \in I\right\}$ of unary predicates. Then $\varphi(x)$ is invariant under bisimulations if and only if it is equivalent to the standard translation of a modal formula.

The proof of the functional completeness part of the theorem (the leftto-right direction) falls outside the scope of this book but can be found in any good textbook on modal logic; see the notes. The other direction of the theorem, which just states that the modal language obeys the design criterion 
(7.1), is the more important one for us here. One way to look at (7.1) is that once we know that a formula is satisfiable at some state in some model, we know by the invariance result that it is also satisfiable in any bisimilar state in any bisimilar model. This means that we can transform the original model into one that suits our purposes best. Obviously, this method applies to any notion of invariance for any language. The nice thing about bisimulation, however, is that it allows the freedom of completely unraveling a model into a tree model.

Definition 7.3.5. Given a model $\mathcal{M}=(W, R, V)$ and a state $s_{1}$ in $\mathcal{M}$, we define the unraveling or unwinding of $\mathcal{M}$ around $s_{1}$ as the following model $\mathcal{M}_{s_{1}}^{u}=\left(\vec{W}_{s_{1}}, \vec{R}, \vec{V}\right)$. Its universe $\vec{W}_{s_{1}}$ is defined as the set of all finite paths through $\mathcal{M}$ starting at $s_{1}$; formally, $\vec{W}_{s_{1}}$ is the collection of all tuples $\left\langle s_{1}, \ldots, s_{n}\right\rangle$ (with $n \geq 1$ ) that satisfy $R s_{i} s_{i+1}$ for all $i<n$. The relation $\vec{R}$ holds of the tuples $\bar{s}=\left\langle s_{1}, \ldots, s_{n}\right\rangle$ and $\bar{t}=\left\langle t_{1}, \ldots, t_{m}\right\rangle$ if and only if $\bar{t}$ is obtained from $\bar{s}$ by adding an $R$-successor of $s_{n}$. Formally, we put $\vec{R} \bar{s} \bar{t}$ if $m=n+1$ and $s_{i}=t_{i}$ for all $1 \leq i \leq n$. Finally, the truth of a proposition letter at a tuple is completely determined by its truth in $\mathcal{M}$ at the last element of the tuple. Formally, let last $\left(\left\langle s_{1}, \ldots, s_{n}\right\rangle\right)$ denote the state $s_{n}$, and define $\vec{V}$ by $\vec{V}(p)=\left\{\bar{s} \in \vec{W}_{s_{1}} \mid \operatorname{last}(\bar{s}) \in V(p)\right\}$.

An example of an unraveling is given in Figure 7.5. Another example can be found in Figure 7.2, in which the model on the lower right-hand side is (an isomorphic copy of) the unraveling of the model on the lower left-hand side.
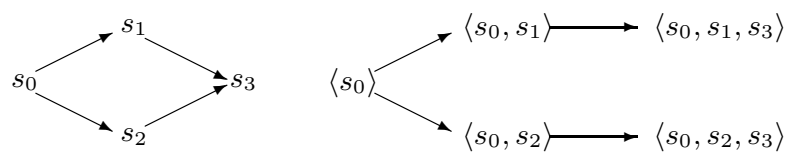

Fig. 7.5. Example of an unraveling

The operation of unraveling is also well known from process theory: the points of the unravelled model $\mathcal{M}_{s_{1}}^{u}$ can be viewed as the process histories or traces that start at $s$. From a technical perspective, the unraveling of $\mathcal{M}$ around $s_{1}$ has certain desirable properties:

- there exists a point with no predecessor, the root;

- $\vec{R}$ is acyclic; and

- $\vec{R}$ is injective in the sense that every point except the root has a unique predecessor.

In other words, the graph $\left(\vec{W}_{s_{1}}, \vec{R}\right)$ is a tree. It is useful to look at this tree as being a normal form of the model.

One can easily check that for any model $\mathcal{M}$ and any state $s$ in $\mathcal{M}$, the (graph of the) function last $: \vec{W}_{s} \rightarrow W$ constitutes a bisimulation between 
$\mathcal{M}_{s}^{u}$ and $\mathcal{M}$ that links $\langle s\rangle$ to $s$. But it immediately follows from this that $\langle s\rangle\left(\right.$ in $\mathcal{M}_{s}^{u}$ ) satisfies exactly the same formulas as $s$ (in $\mathcal{M}$ ). Combining this with the observation that unravelings are trees, we find that every satisfiable basic modal formula is also satisfiable in a tree. We say that a modal system $(\mathcal{L}, \mathrm{K}, \Vdash)$ has the tree model property if, for every satisfiable formula $\xi$ in $\mathcal{L}$, there exists a tree in $\mathrm{K}$ in which $\xi$ is satisfiable. Thus we have established the following.

Theorem 7.3.6. The basic modal system has the tree model property.

The following observation is a driving force behind our search for decidable modal fragments of first-order logic.

Observation 2. In proving the last theorem we did not use any property of basic modal logic other than its invariance under bisimulation. This means that, in fact, any modal system with a bisimulation-invariant semantics has the tree model property - provided, of course, that tree models are admissible in the system!

\section{Games}

In order to facilitate a comparison with the Ehrenfeucht-Fraïssé games often used in first-order logic, it is convenient to rephrase the notion of a bisimulation between two models in game-theoretic terms. Let $\mathcal{M}=(W, R, V)$ and $\mathcal{M}^{\prime}=\left(W^{\prime}, R^{\prime}, V^{\prime}\right)$ be two models, and let $s_{0}$ and $s_{0}^{\prime}$ be two states in $\mathcal{M}$ and $\mathcal{M}^{\prime}$, respectively.

We define the bisimulation game as a variant of the familiar EhrenfeuchtFraïssé games. In each round of the bisimulation game, $\forall$ selects one of the two models and, inside this model, he chooses a successor of the element played in the previous round (in the first round he chooses a successor of $s_{0}$ or $s_{0}^{\prime}$ ). $\exists$ responds with a successor of the last element played in the other structure. The length $l$ of the game is defined as the number of rounds and can be either finite or infinite. A match of the game thus gives rise to two sequences: $\bar{s}=s_{0}, s_{1}, s_{2}, \cdots$ and $\bar{s}^{\prime}=s_{0}^{\prime}, s_{1}^{\prime}, s_{2}^{\prime}, \cdots$, of elements in $\mathcal{M}$ and $\mathcal{M}^{\prime}$, respectively.

$\exists$ wins this match of the game if for each $i, s_{i}$ and $s_{i}^{\prime}$ agree on the truth of all propositional variables; otherwise, $\forall$ wins. We say that $\exists$ has a winning strategy in the game $\mathcal{G}_{b}^{l}\left(\mathcal{M}, \mathcal{M}^{\prime}, s, s^{\prime}\right)$ of $l$ rounds played in $\mathcal{M}, s, \mathcal{M}^{\prime}, s^{\prime}$ if $\exists$ can win every match of length at most $l$ starting in the states $s$ and $s^{\prime}$.

It should be fairly clear that $\forall$ is trying to spoil a bisimulation between $\mathcal{M}, s$ and $\mathcal{M}^{\prime}, s^{\prime}$, while $\exists$ has the opposite intention. Indeed we have a precise game-theoretic characterization of the notion of bisimulation.

Proposition 7.3.7. There exists a bisimulation between $\mathcal{M}, s$ and $\mathcal{M}^{\prime}, s^{\prime}$ if and only if $\exists$ has a winning strategy in the game $\mathcal{G}_{b}^{\omega}\left(\mathcal{M}, \mathcal{M}^{\prime}, s, s^{\prime}\right)$. 
As a corollary, we find that the truth of modal formulas is preserved when $\exists$ has a winning strategy in the game $\mathcal{G}_{b}^{\omega}\left(\mathcal{M}, \mathcal{M}^{\prime}, s, s^{\prime}\right)$. What is interesting about these games is that they facilitate a more fine-grained perspective on such connections. In particular, if $\exists$ has a winning strategy in a game of fixed finite length $n$, what can we say about the preservation of modal formulas? Quite a lot, according to the proposition below; in order to formulate it, we need the notion of the modal depth of a formula - the straightforward analog of first-order logic's quantifier depth.

Definition 7.3.8. The modal depth $d_{\diamond}(\varphi)$ of a modal formula is inductively defined as follows:

$$
\begin{aligned}
d_{\diamond}(p) & =0 \\
d_{\diamond}(\neg \psi) & =d_{\diamond}(\psi) \\
d_{\diamond}\left(\psi_{1} \wedge \psi_{2}\right) & =\max \left(d_{\diamond}\left(\psi_{1}\right), d_{\diamond}\left(\psi_{2}\right)\right) \\
d_{\diamond}(\diamond \psi) & =1+d_{\diamond}(\psi) .
\end{aligned}
$$

Proposition 7.3.9. Let $\Phi$ be a finite set of proposition letters, and let $n$ be some natural number. The following are then equivalent:

1. $\exists$ has a winning strategy in the game $\mathcal{G}_{b}^{n}\left(\mathcal{M}, \mathcal{M}^{\prime}, s, s^{\prime}\right)$.

2. $\mathcal{M}, s$ and $\mathcal{M}^{\prime}, s^{\prime}$ satisfy the same $\mathcal{L}_{\diamond}(\Phi)$ formulas up to modal depth $n$.

We omit the fairly standard proof of this proposition - the notes contain references. The analogous proposition relating $\mathcal{G}_{b}^{\omega}\left(\mathcal{M}, \mathcal{M}^{\prime}, s, s^{\prime}\right)$ and the full modal language does not hold, as is witnessed by the models in Figure 7.3.

What have we gained from this slightly more fine-grained analysis of modal logic? We have already seen that any satisfiable modal formula $\xi$ can also be satisfied at the root $s$ of a tree model $\mathcal{M}$ : take, for instance, some unraveling of the original model. From Proposition 7.3.9 we can conclude that such a $\xi$ can also be satisfied in a bounded-depth tree model $\mathcal{M}^{\prime}$ : simply take the tree model $\mathcal{M}$ and throw away all states that are further than $d$ steps away from the root $s$ (where $d$ is the modal depth of $\xi$ ). It is obvious that $\exists$ has a winning strategy in the bisimulation game $\mathcal{G}_{b}^{d}\left(\mathcal{M}, \mathcal{M}^{\prime}, s, s\right)$. From this it follows that $\xi$ holds at the root $s$ of the bounded-depth tree $\mathcal{M}^{\prime}$.

\section{Finite trees}

We shall now show that the satisfiability problem for the basic modal language (with respect to the class of all models) is indeed decidable. We shall do so by establishing the bounded model property. A modal language is said to have this property with respect to a class $\mathrm{K}$ of models if every formula $\xi$ that is satisfiable in some model in $\mathrm{K}$ can in fact be satisfied in a finite model in $\mathrm{K}$ of bounded size (that is, the size of the model is bounded by some computable function on $|\xi|)$. 
Now, in order to show that the language of basic model logic has this bounded model property, let $\xi$ be an arbitrary satisfiable formula. We have already seen that we may assume that $\xi$ is true at the root of a tree model $\mathcal{M}$ of depth not exceeding the modal depth of $\xi$. This tree model might still be infinite because of infinite branching, but now we recursively prune the tree as follows. Starting at the root, for every subformula of $\xi$ of the form $\diamond \varphi$, we choose a successor of the root at which $\varphi$ is true (if such a successor exists at all). Obviously, at most $b$ successors can be chosen, where $b$ is the number of diamond subformulas of $\xi$. Hence, by deleting from the model all successors that have not been chosen, together with their descendants, we obtain a tree model whose branching degree at the root is at most $b$. A simple verification shows that $\xi$ still holds at the root. Now we repeat this process at each of the chosen successors of the root and continue until the leaves of the tree are reached. Obviously, $\xi$ is still satisfied at the root.

Thus we have proved the following.

Proposition 7.3.10. Any satisfiable modal formula $\xi$ can be satisfied at the root of a finite tree model, of which the depth is bounded by the modal depth of $\xi$ and the branching degree is bounded by the number of diamond subformulas of $\xi$.

The decidability of basic modal logic is a straightforward corollary of this. (For instance, we can use the standard translation, the equivalence (7.3), and the fact that first-order model checking is decidable.)

Theorem 7.3.11. The basic modal system $\mathbf{K}$ has a decidable satisfiability problem.

We shall now describe a decision procedure which on input $\xi$ systematically tries to build the tree model for $\xi$ described in Proposition 7.3.10. The procedure is based on a simple but powerful idea: it tries to build a model $(W, R, V)$ in which

1. the states are finite sets $\Delta$ of "relevant" formulas;

2. we have the following "truth = membership" principle:

$$
\varphi \in \Delta \leftrightarrow \mathcal{M}, \Delta \Vdash \varphi
$$

for all relevant formulas $\varphi$ and for all states $\Delta$ in the model.

Suppose we can build such a model for an input formula $\xi$. Then by the truth lemma (7.4), $\xi$ is satisfiable if it belongs to some state $\Delta$ in our model. Our implementation of the decision procedure is based purely on the proof of the truth lemma; hence, in order to motivate the procedure, we now indicate how to arrive at this proof. Fix a formula $\xi$.

Let us first confine the collection of relevant formulas. This set will change from state to state but, basically, all relevant formulas will be subformulas of $\xi$; we need a little extra, though. Given a formula $\varphi$, let $\sim \varphi$ denote the 
formula $\psi$ if $\varphi$ is of the form $\neg \psi$; otherwise, $\sim \varphi$ is the formula $\neg \varphi$; we say that a set $\Sigma$ of formulas is closed under taking single negations if $\sim \varphi \in \Sigma$ whenever $\varphi \in \Sigma$. This notion enables us to pretend that a finite set is closed under taking negations by treating $\sim \varphi$ as if it were the real negation of $\varphi$. Now, given a set of formulas $\Sigma$, let $C l(\Sigma)$ be the smallest set of formulas that extends $\Sigma$ and is closed under taking subformulas and single negations. When $\xi$ is a formula, we denote the set $C l(\{\xi\})$ of relevant $\xi$ formulas as $C l(\xi)$; it is easy to see that the cardinality of $C l(\xi)$ is linear in the length of $\xi$.

Each state of our model $\mathcal{M}=(W, R, V)$ will be a subset of $C l(\xi)$, but rather than precisely define the universe $W$ now, we assume here that we have defined it, and gather sufficient requirements to be placed on this definition to enable a proof of (7.4). First we consider the valuation: the truth lemma prescribes a unique way to define $V$, at least for the proposition letters occurring in $\xi: V(p)=\{\Delta \mid p \in \Delta\}$. For a definition of the relation $R$, we shall be rather opportunistic. Again we use the truth lemma as our guideline: it shows that if $R\left(\Delta, \Delta^{\prime}\right)$ is to hold, then we should avoid the existence of a relevant formula $\diamond \varphi$ such that $\varphi \in \Delta^{\prime}$ but $\diamond \varphi \notin \Delta$. Now, $R$ is defined by turning this requirement into a definition: we put $\left(\Delta, \Delta^{\prime}\right)$ into $R$ precisely when the above situation does not occur.

Let us now see what requirements we have to impose on the set $W$; that is, suppose that we want to give an inductive proof of the "truth = membership" principle. Assume that with each state $\Delta$, we have associated a collection $\Sigma \supseteq \Delta$ of relevant formulas.

Obviously, the atomic case of the truth lemma holds by the definition of $V$. For the inductive boolean cases to go through, it is sufficient to require that $\Delta$ is maximal with respect to being a propositionally consistent subset of $\Sigma$. That is, $\Delta$ and $\Sigma$ have to satisfy the following condition $\operatorname{Prop}-\operatorname{Max}(\Delta, \Sigma)$ : - (for each $\sim \varphi \in \Sigma): \sim \varphi \in \Delta \leftrightarrow \varphi \notin \Delta$; and

- (for each $\varphi \wedge \psi \in \Sigma): \varphi \wedge \psi \in \Delta \leftrightarrow \varphi \in \Delta$ and $\psi \in \Delta$.

The inductive modal case imposes two further constraints, one for each direction of the truth lemma. We met the first one already when we defined our relation $R$, but given that $\operatorname{Prop}-\operatorname{Max}(\Delta, \Sigma)$ holds, we can reformulate this condition as follows:

- if $R\left(\Delta, \Delta^{\prime}\right)$, then for all $\diamond \psi \in \Sigma: \sim \diamond \psi \in \Delta$ implies $\sim \psi \in \Delta^{\prime}$.

This formulation clearly brings about the conditions that each successor of $\Delta$ should satisfy. The other direction of the truth lemma for the case $\varphi=\diamond \psi$ presents an existential requirement:

- if $\diamond \psi \in \Delta$, then there has to be a $\Delta^{\prime}$ such that $\psi \in \Delta^{\prime}$ and $R\left(\Delta, \Delta^{\prime}\right)$.

Observe that in this last existential requirement we encounter the branchcutting argument that we saw earlier on. Then we only kept successor states if there was a reason in the form of a $\diamond \psi$ formula; now, we only create a successor if we need it as a witness for such a formula. The search for suitable successors is the driving force behind our algorithm. 
But what about these associated sets of relevant formulas? Will every formula in $C l(\xi)$ be relevant throughout the procedure? No, and this is precisely what will bound the recursion depth of the algorithm: the set of relevant formulas will decrease as we move away from the root of the model. This is reminiscent of the bounded depth of the tree model in Proposition 7.3.10. In particular, the set of relevant formulas for a state $\Delta$ which is $m$ steps away from the root will consist of all formulas from $C l(\xi)$ of modal depth at most $d_{\diamond}(\xi)-m$.

The algorithm presented in Figure 7.6 implements this search for a tree model. We claim that for sets of formulas $\Delta$ and $\Sigma$ such that $\Sigma$ is closed under taking subformulas and single negations, $K$-World $(\Delta, \Sigma)$ will be true iff there exists a tree model $\mathcal{M}$ such that at the root $s$, for all $\psi \in \Sigma,(\mathcal{M}, s \Vdash$ $\psi \leftrightarrow \psi \in \Delta)$. This function can be used to solve the satisfiability problem for the basic modal system, since $\xi$ is satisfiable iff there exists a set $\Delta \subseteq C l(\xi)$ such that $\xi \in \Delta$ and $K$ - $\operatorname{World}(\Delta, C l(\xi))$ is true.

Note that with each recursive call of $K$-World, the size of the set $\Sigma$ decreases, since we include formulas of smaller modal depth only. Thus the recursion depth is bounded by the modal depth of the input formula $\xi$. That the function is correct can be proved by induction on the size of $\Sigma$; we leave this to the reader. By an appeal to Savitch's Theorem (PSPACE = NPSPACE), it is not hard to see that the procedure runs in PSPACE. We shall come back to this aspect in Section 7.5.

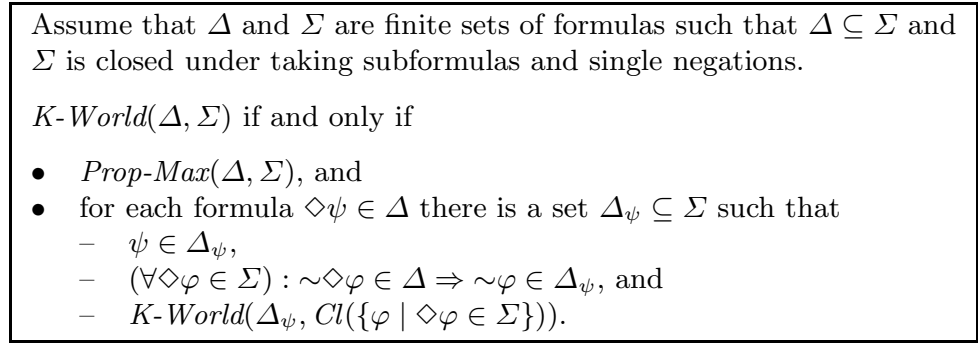

Fig. 7.6. The function $K$-World decides $\mathbf{K}$ satisfiability.

This finishes the proof of Theorem 7.3.11; in the remainder of this chapter we shall analyze this proof and see how much of it can be used for other (modal) logics.

\section{Looseness and locality}

In analyzing this decidability proof, we can distinguish a number of relevant properties of the basic modal system. First of all, bismulation invariance ensures that, in order to check the satisfiability of a modal formula, we only 
have to worry about "loose" tree models. We shall call this the looseness principle of basic modal logic; this property has recently gained status as either the single or at least the crucial property that makes modal logic so robustly decidable.

However, we believe that looseness is not all there is to say in relation to explaining the decidability (or low complexity) of the basic modal system $\mathbf{K}$. The semantics of the basic modal language shows that modal formulas only have a limited access to the model. This is what we dub the locality principle of modal logic, and one can make this rather vague notion precise in (at least) two ways.

First, in the above proof we used the fact that the effect of a modal formula is bounded by its modal depth. In particular, when working in a tree model we can prune the relevant neighborhood of a state even further by the method of selecting witnesses for $\diamond$-subformulas. All in all, we find that in order to check whether a modal formula holds at a given state of some tree model, one only has to worry about a bounded, "local" part of the tree model. In particular, what the basic modal language does not have is global expressive power. We say that a modal system $(\mathcal{L}, \mathrm{K}, \Vdash)$ has global expressive power if it can define the universal diamond $\mathrm{E}$; that is, if there is a formula $\varphi(p)$ such that for every model $\mathcal{M}$ in $\mathrm{K}$ and every state $s$ in $\mathcal{M}$, we have

$$
\mathcal{M}, s \Vdash \varphi(p) \leftrightarrow \mathcal{M}, t \Vdash p \text { for some } t \text { in } \mathcal{M} \text {. }
$$

By the first locality principle, we mean the lack of global expressive power. Later on, we shall see that if we add even the tiniest bit of global expressive power to the basic modal system, we destroy its finite tree property and lift the complexity of the satisfiability problem from PSPACE to EXPTIME.

We have already met the second locality principle in Observation 1. From the fact that the basic modal language belongs to the two-variable fragment $\mathrm{FO}_{2}$ of first-order logic, we may conclude that the satisfiability problem for the basic modal system can be reduced to that for $\mathrm{FO}_{2}$. But, for every modal language in which the connectives have a first-order truth definition, we can come up with a "standard translation", so if the language has only finitely many connectives, this standard translation remains within a fixed finitevariable fragment. Hence, if we consider a modal system $(\mathcal{L}, \mathrm{K}, \Vdash)$ in which $\mathcal{L}$ has only finitely many connectives and in addition, the class of models $\mathrm{K}$ allows a definition in some finite-variable fragment, then the satisfiability problem for the modal system can be reduced to that of some finite-variable fragment $F O_{k}$. Why are we interested so much in these fixed finite-variable fragments? As we shall see later, one reason is that they have tractable model checking problems, whereas the full first-order language does not. To be concrete, given a finite first-order model $\mathcal{M}$ and a first-order sentence $\xi$, the problem of whether $\mathcal{M} \models$ $\xi$ is decidable in PTIME in $\mathcal{M}$ and in $\xi$, if $\xi$ is from a fixed-variable fragment, whereas it is in PSPACE if $\xi$ is an arbitrary first-order sentence. With the second locality principle, we shall mean this reducibility of the satisfiability problem to the satisfiability of some fixed finite-variable fragment of first-order 
logic (or perhaps of some higher-order formalism, as in the case of $\mathbf{K}^{*}$ which we shall discuss later on).

It will be useful later to state what we mean by looseness and locality in terms of the bisimulation game. Since it is $\forall$ who tries to spoil a bisimulation, the strength of the bisimulation relation is determined by the moves $\forall$ is allowed to make. Indeed, $\forall$ 's powers are limited. First, observe that although a match is made up of long sequences of pairs of states, after every round it is only the last pair which is important. Given such a pair, $\forall$ is allowed to choose a new element, but only if it is a successor of a state in the pair. $\exists$ replies and then the players check whether the match is over because $\forall$ has won or not. If not, the previous pair "is deleted from memory" and the game continues.

We could view the game as being played by moving two windows across the models. These windows completely hide the model from view, except for at most two states. Both players move the windows across the models, and $\forall$ has the initiative. Now, the principle of looseness means that the states which are visible through the window are always connected by the accessibility relation (this shows we could equally well have dubbed the "looseness principle a "locality" principle as well). The second locality principle is embodied in the fixed finite dimension of the window. (To describe the first locality principle, games do not seem to be the optimal way.)

Summarizing, it seems that these looseness and locality principles in tandem cause the decidability of the basic modal system: looseness means that one only has to check trees, and the first locality principle adds that in fact finite trees suffice. (For the contribution of the second locality principle, the reader will have to wait until we discuss the generalization of the modal language to the guarded and packed fragments of first-order logic in section 7.6.) There can be no doubt that looseness is the most important property for the decidability of a modal system; in fact, if we confine ourselves to the class $\mathrm{M}$ of all modal models, it will be hard to find a bisimulation-invariant system with an undecidable satisfiability problem! The reason for this is that the modal mu-calculus is decidable, and this modal system can be characterized as the bisimulation-invariant fragment of monadic second-order logic over a signature of binary relations.

\subsubsection{Notes}

Recent years have seen a proliferation of modern textbooks on modal logics, of which we mention those by Chagrov \& Zakharyaschev [14], Popkorn [49] and Blackburn, de Rijke, \& Venema [9].

The standard translation, in various forms, can be found in the work of a number of writers on modal and tense logic in the 1960s. Van Benthem [4] first made clear the importance of systematic use of the standard translation to access results and techniques from classical modal theory. The observation 
that at most two variables are needed to translate basic modal formulas into first-order logic is due to Gabbay [20]. The earliest systematic study of finitevariable fragments seems to be due to Henkin [26] in the setting of algebraic logic, while Immerman \& Kozen [34] studied the link with complexity and database theory. See Otto [47] for more on finite-variable logics, or Marx \& Venema [43] for a modal perspective on these logics.

Bisimulations were first introduced (under a different name) by van Benthem $[4,5]$. The notion was introduced independently in computer science, as an equivalence relation on process graphs; the first reference seems to be Park [48], while the classic computer science paper on the subject is Hennessy \& Milner [28]; the latter paper also discusses finitary approximations to bisimulations. The notion of unraveling a modal model stems from Dummett \& Lemon [16]. Proposition 7.3.9 is analogous to similar characterizaions of logical equivalence for first-order logic, due to Ehrenfeucht and Fraïssé (see [31]).

Theorem 7.3.4, the Characterization Theorem which identifies modal logic as the bisimulation-invariant fragment of first-order logic, is due to van Benthem $[4,6]$. The back-and-forth clauses of a bisimulation can be adapted to analyze the expressivity of a wide range of modal logics, and such analyses are now commonplace. For instance, Janin \& Walukiewicz [35] have proved that Kozen's modal mu-calculs is the bisimulation-invariant fragment of a natural monadic second-order logic over process graphs. Related model-theoretic characterizations can be found in Immerman \& Kozen [34] (for finite-variable logics). Rosen [51] has presented a version of the Characterization Theorem that also works for the case of finite models.

Finite models have long been used to establish decidability, both in modal logic and elsewhere. Arguments based on finite axiomatizability together with the finite model property can be traced back to Harrop [25]. The computational complexity of the satisfiability problem for the basic modal system was established by Ladner [37]: it is PSPACE-complete. The function $K$-World is a slight variation of Ladner's procedure. The presentation given here is taken from Spaan [55].

The problem of whether $\mathcal{M} \models \xi$ for a given a finite first-order model $\mathcal{M}$ and a first-order sentence $\xi$, is PTIME-complete when $\xi$ is from a fixed finitevariable fragment (see Immerman [33], Vardi [57]), but PSPACE-complete when $\xi$ is an arbitrary first-order sentence (Chandra \& Merlin [15]).

\subsection{Some Variations}

In this section, we shall consider some modal systems that are variations on the basic modal system. Apart from our wish to introduce some new proof techniques for establishing decidability of a modal system, such as the filtration and mosaic methods, our aim in this section is to clarify the looseness and locality principles that we have just introduced. 
We shall first investigate some modal systems that are fairly "tight" in the sense that their class of models is based on grid-like structures; as we shall see, such a lack of looseness brings these systems close to the danger zone of undecidability. Nevertheless, if the locality principles still hold, decidability is still possible. The second system that we consider is obtained by adding just a grain of global expressive power to the basic modal language, while keeping the looseness condition. We shall see that the system is still decidable, but it no longer has the finite-tree property. Finally, we consider a modal system in which the operator is not bisimulation-invariant at all; however, as we shall see, it does have another kind of looseness property, and this enables us to prove its decidability.

\subsubsection{Neither locality nor looseness: grid logics}

In this subsection, we consider modal systems that cannot be called loose or local. We shall first meet a simple modal system that is tailored towards encoding the $\mathbb{N} \times \mathbb{N}$-tiling problem, and is undecidable; as a contrast, we shall also discuss a second system with grid-like models which has a decidable satisfiability problem.

\section{A tiling logic}

In looking for the opposite of looseness one is bound to end up with a grid. Grids are well known in complexity theory, since they play an important role in the formulation of a class of complete problems for various complexity classes: tiling problems. A tile is a one-by-one square which has a "color" on each of its sides; these colors are given by four functions "right", "left", "up", and "down". Given a set $T$ of tiles, a tiling of the grid $\mathbb{N} \times \mathbb{N}$ by $T$ is a map $t$ from $\mathbb{N} \times \mathbb{N}$ to $T$ satisfying, for all $n, m \in \mathbb{N}$,

$$
\begin{aligned}
& \operatorname{right}(t(n, m))=\operatorname{left}(t(n+1, m)), \\
& \operatorname{up}(t(n, m))=\operatorname{down}(t(n, m+1)) .
\end{aligned}
$$

Tiles are assumed to be fixed in orientation, so the above conditions say that colors of adjacent tiles match. (We note that it is not necessary to use all tiles of $T$ in a tiling of $\mathbb{N} \times \mathbb{N}$.) If such a tiling exists, we say that $T$ can tile $\mathbb{N} \times \mathbb{N}$.

The following problem is undecidable:

$\mathbb{N} \times \mathbb{N}$ tiling: Given a finite set $T$ of tiles, can $T$ tile $\mathbb{N} \times \mathbb{N}$ ?

We shall now define a modal system Tile which is tailored to encode the above tiling problem. The language of Tile contains two unary modalities $\diamond_{r}$ and $\diamond_{u}$ plus the universal modality $\mathrm{E}$. In a model of the form $\left(W, R_{r}, R_{u}, V\right)$, these modalities receive their meaning in the usual way: 


$$
\begin{aligned}
& \mathcal{M}, s \Vdash \diamond_{r} \varphi \leftrightarrow \mathcal{M}, t \Vdash \varphi \text { for some } t \text { with } R_{r} s t, \\
& \mathcal{M}, x \Vdash \diamond_{u} \varphi \leftrightarrow \mathcal{M}, t \Vdash \varphi \text { for some } t \text { with } R_{u} s t, \\
& \mathcal{M}, x \Vdash \mathrm{E} \varphi \leftrightarrow \mathcal{M}, t \Vdash \varphi \text { for some } t .
\end{aligned}
$$

In the intended class of grid models, $R_{r}$ and $R_{u}$ are (the graphs of) two commuting total functions. In particular, grid models satisfy the following condition:

$$
\forall x y z\left(\left(R_{r} x y \wedge R_{u} x z\right) \rightarrow \exists w\left(R_{r} z w \wedge R_{u} y w\right)\right) .
$$

Because of this, the class of grid models is not closed under unraveling; hence, Tile does not satisfy the looseness principle. It is also rather obvious that the first locality principle fails as well, in the presence of the universal modality; we leave it to the reader to verify that the class of grid models can be defined using three variables only, and Tile thus satisfies the second locality principle.

Theorem 7.4.1. The satisfiability problem of Tile is undecidable.

Proof. Obviously, we reduce the $\mathbb{N} \times \mathbb{N}$-tiling problem to the satisfiability problem for Tile. We present a procedure that outputs, for every instance $T$ of the tiling problem, a formula $\varphi_{T}$ such that

$$
\mathrm{A} \varphi_{T} \text { is Tile-satisfiable iff } T \text { can tile } \mathbb{N} \times \mathbb{N} \text {. }
$$

(Recall that $A$ is the box version of $E$; that is, $A \varphi$ abbreviates $\neg \mathrm{E} \neg \varphi$.)

Take, for any set $T=\left\{T_{1}, \ldots, T_{k}\right\}$ of tiles, a corresponding set $\left\{t_{1}, \ldots, t_{k}\right\}$ of propositional variables. Define $\varphi_{T}$ as the conjunction of the following formulas (where $i$ ranges over $1, \ldots, k)$ :

(A1) $\bigvee_{1 \leq i \leq k} t_{i}$

$\left(A 2_{i}\right) t_{i} \rightarrow \bigwedge_{i \neq j} \neg t_{j}$

$\left(A 3_{i}\right) t_{i} \rightarrow \diamond_{r} \bigvee\left\{t_{j} \mid \operatorname{right}\left(T_{i}\right)=\operatorname{left}\left(T_{j}\right)\right\}$

$\left(A 4_{i}\right) t_{i} \rightarrow \diamond_{u} \bigvee\left\{t_{j} \mid \operatorname{up}\left(T_{i}\right)=\operatorname{down}\left(T_{j}\right)\right\}$

It follows almost immediately that $T$ tiles $\mathbb{N} \times \mathbb{N}$ if and only if there exists a Tile model where $\varphi_{T}$ holds throughout. (The reader should verify that in the proof of the left-to-right direction of (7.6) the property (7.5) of grid models is crucial.) This, in turn, is equivalent to the formula $A \varphi_{T}$ being satisfiable in some Tile model. Thus (7.6) holds and we have reduced the undecidable tiling problem to the Tile satisfiability problem.

We hasten to remark that the undecidability of this system has nothing to do with the fact that we are dealing with more than one modality here; one can easily transform this example into an undecidable modal system in the basic modal language extended with the universal modality, or in the basic modal language proper.

It is interesting to note that without the universal access to the models provided by $A$, these grid logics become quite harmless. In fact, their grid-like nature ensures that every satisfiable formula $\xi$ is satisfiable in a model whose size is at most $|\xi|^{2}+1$. 
Theorem 7.4.2. Let Tile $^{-}$be the modal system Tile, but now without the universal modality. Then every Tile $^{-}$-satisfiable formula $\xi$ is satisfiable in a $\mathrm{Tile}^{-}$model of size at most $|\xi|^{2}+1$. As a corollary, Tile ${ }^{-}$has a decidable satisfiability problem.

Proof. Let $\mathcal{M}$ satisfy $\xi$ at $s$. Let $k$ be the modal depth of $\xi$, we then have that $k \leq|\xi|$. By Proposition 7.3.9, $\xi$ is still satisfiable in the model $\mathcal{M}^{\prime}$, defined as the substructure of $\mathcal{M}$ with universe $s$ together with all states reachable in at most $k\left(R_{r^{-}}\right.$or $\left.R_{u^{-}}\right)$steps from $s$. Clearly, the size of the universe of $\mathcal{M}^{\prime}$ is at most $k^{2}$. Unfortunately, $\mathcal{M}^{\prime}$ is not a Tile model, because not every state has an $R_{r}$ and $R_{u}$ successor. In order to mend this, we add one dummy state $x$ to the universe of $\mathcal{M}^{\prime}$ and put a link from $w$ to $x$ for all states $w$ (including $x$ itself) that do not have a successor yet. That is, we define $W^{-}=W^{\prime} \cup\{x\}$ and $R_{r}^{-}=R_{r}^{\prime} \cup\left\{(w, x) \mid R_{r}^{\prime} w y\right.$ for no $y$ in $\left.\mathcal{M}^{\prime}\right\}$, and likewise for $R_{u}^{-}$. Let the valuation stay the same, i.e., we define $V^{-}(p)=V^{\prime}(p)$ for all $p$.

The resulting model $\mathcal{M}^{-}$is a Tile model. Clearly, $\xi$ is still satisfied at $s$ in this new model, since $x$ is "too far away" to have any effect on the truth of $\xi$. This proves the first part of the theorem. Decidability now follows because it is decidable whether a finite model is a Tile model.

\section{$\mathrm{S}^{2}$}

The second logic that we consider here is also based on grid-like structures, but here we require only that the models are two-dimensional in nature; there will be no orderings or functions around. The language has two diamonds, $\diamond_{0}$ and $\diamond_{1}$, with the standard truth definition. The models are of the form $\mathcal{M}=\left(W, \equiv_{0}, \equiv_{1}, V\right)$, where we require that $\left(W, \equiv_{0}, \equiv_{1}\right)$ is in fact a square over some set $U$. That is, $W$ consists of the set $U \times U$ of all pairs over $U$, and $s \equiv_{i} t$ holds if $s_{i}=t_{i}$ : the $i$ th coordinate of $s$ and the $i$ th coordinate of $t$ should be the same. We denote the resulting system by $\mathbf{S} \mathbf{5}^{\mathbf{2}}$.

As a modal system, $\mathbf{S 5 ^ { 2 }}$ might look rather obscure, but as a logic, it is well known. In fact, it is the exact modal counterpart of a restricted fragment of first-order logic with two variables in a signature that has a binary relation symbol $R$ for every propositional variable $r$. This can be seen as follows. First, observe that the $\mathbf{S} 5^{2}$ model $\mathcal{M}=\left(W, \equiv_{0}, \equiv_{1}, V\right)$ with $W=U \times U$ is uniquely determined by the first-order model $(U, V)$ for the signature described. Also observe that we may identify assignments $s$ mapping the two variables $x_{0}$ and $x_{1}$ to $U$ with pairs $\left(s\left(x_{1}\right), s\left(x_{0}\right)\right) \in W$. Thus, viewing the states of the modal models as assignments, we may read the statement " $\varphi$ holds in $(U, V)$ under assignment $s$ " modally as "in model $\left(U \times U, \equiv_{0}, \equiv_{1}, V\right), \varphi$ is true at state $s$ ". Because $\mathbf{S 5}^{\mathbf{2}}$ models are squares, the truth definition of the diamonds can be rewritten exactly as the definition of the first-order existential quantifiers:

$$
\mathcal{M},(a, b) \Vdash \diamond_{1} \varphi \leftrightarrow \text { there exists } a^{\prime} \text { such that } \mathcal{M},\left(a^{\prime}, b\right) \Vdash \varphi \text {. }
$$


Thus $\diamond_{i}$ is another way of writing $\exists x_{i}$. In a similar way, one can define modal systems $\mathbf{S 5}^{\mathbf{n}}$ corresponding to first-order logic with $n$ variables for any $n$. See the notes for references.

It will be obvious that this class of models is not closed under unraveling, and that $\mathbf{S 5}^{\mathbf{2}}$ will not have the tree model property. Concerning the locality principles, observe that this system has full global expressive power: the "combined" operator $\diamond_{0} \diamond_{1}$ behaves just like the universal diamond E. Nevertheless, the system is decidable, and a proof of this uses some kind of finite model property as well.

Here, instead of defining a finite model for $\xi$ by selecting points out of the old model, we shall identify points in the big model and define the finite model as some sort of quotient structure, which we call a filtration of the original model. It will turn out that this filtration will not be a square itself but a square-like structure, which we dub a pseudo-square here. That is to say, in the underlying frame $\left(W, R_{0}, R_{1}\right)$ both $R_{0}$ and $R_{1}$ are equivalence relations, and their composition should be the universal relation. That is, $\left(W, R_{0}, R_{1}\right)$ has to validate

$$
\forall x y \exists z\left(R_{0} x z \wedge R_{1} z y\right) .
$$

For these kind of structures, we can prove the following proposition, which establishes the bounded finite model property of the language with respect to the class of pseudo-squares. (In fact, the system does have the bounded finite model property, but this is much harder to establish.) As we saw before, decidability follows immediately, because it is decidable whether a finite structure is a pseudo-square.

Proposition 7.4.3. Any $\mathbf{S 5}^{\mathbf{2}}$-formula $\xi$ is satisfiable in a square iff it is satisfiable in a pseudo-square of size not exceeding $2^{|\xi|}$. As a consequence, $\mathbf{S 5}^{\mathbf{2}}$ has a decidable satisfiability problem.

Proof. We shall concentrate on the left-to-right direction of this proof, since we are interested only in explaining the notion of filtration at the moment. (For the other direction of the proof, one shows that given a pseudo-square model, one can always find a square that is bisimilar to it - in fact, bisimilar through a functional bisimulation; see the notes.)

Suppose $\xi$ is satisfied somewhere in the square model $\mathcal{M}=\left(W, \equiv_{0}, \equiv_{1}, V\right)$. From this we shall prove that $\xi$ is true somewhere in a filtration $\mathcal{M}^{f}$ of $\mathcal{M}$. As we have mentioned already, filtrating a model means collapsing it. But when will two points in the original model be identified? Generally, taking a quotient of a structure means identifying points without "relevant" differences; in the present context this can be interpreted as "satisfying the same subformulas of $\xi$ ". Formally, we define $\mathrm{Cl}(\xi)$ to be the smallest set of formulas containing $\xi$ which is closed under subformulas. Now, we define the following relation on $W$ :

$$
s \sim s^{\prime} \leftrightarrow \text { for all } \varphi \text { in } C l(\xi): \mathcal{M}, s \Vdash \varphi \text { iff } \mathcal{M}, s^{\prime} \Vdash \varphi
$$


Obviously, $\sim$ is an equivalence relation. Our filtrated model will be based on the equivalence classes of this relation, and so we introduce some notation: by $\bar{s}$ we denote the equivalence class of a point $s$, and by $W^{f}$, the set of these classes. Note that $\left|W^{f}\right| \leq 2^{|\xi|}$ as $|C l(\xi)|$ is bounded by $|\xi|$.

What would be a good definition for the relations $R_{0}$ and $R_{1}$ on $W^{f}$ ? In general, this is where the filtration method needs some creative input. Now, if the only requirement were that $\xi$ were to be true somewhere in the resulting model, there would be a whole family of definitions that work (in the sense that they ensure (7.8) below). But the extra constraint, namely that the resulting model should be a pseudo-square, imposes some extra restrictions. Nevertheless, the following definition works:

$$
R_{i} \bar{s} \bar{t} \text { if for all } \diamond_{i} \varphi \in C l(\xi): \mathcal{M}, s \Vdash \diamond_{i} \varphi \text { iff } \mathcal{M}, s^{\prime} \Vdash \diamond_{i} \varphi \text {. }
$$

(Observe that this is well defined, by the fact that $\sim$-equivalent points agree about all formulas in $C l(\xi)$.) Finally, the definition of $V^{f}$ is rather obvious:

$$
V^{f}(p)=\left\{\bar{s} \in W^{f} \mid s \in V(p)\right\} .
$$

Note that this is well defined for all proposition letters $p$ occurring in $\xi$.

We can prove the main claim concerning filtration:

$$
\text { for all formulas } \varphi \in C l(\xi): \mathcal{M}, s \Vdash \varphi \text { iff } \mathcal{M}^{f}, \bar{s} \Vdash \varphi \text {. }
$$

This claim is proved by a formula induction. Leaving the straightforward induction base and the boolean cases of the inductive step to the reader, we concentrate on the case where $\varphi$ is of the form $\diamond_{0} \psi$. (The case where $\varphi$ is of the form $\diamond_{1} \psi$ is of course completely analogous.)

First, assume that $\mathcal{M}, s \Vdash \diamond_{0} \psi$. Then, by definition, there is some $s^{\prime}$ in $\mathcal{M}$ such that $s \equiv_{0} s^{\prime}$ and $\mathcal{M}, s^{\prime} \Vdash \psi$. By the inductive hypothesis, this gives that $\mathcal{M}^{f}, \bar{s}^{\prime} \Vdash \psi$. It easily follows from the definitions that $s \equiv_{0} s^{\prime}$ implies $R_{0} \bar{s} \bar{s}^{\prime}$. But then it follows immediately that $\mathcal{M}^{f}, \bar{s} \Vdash \diamond_{0} \psi$. For the other direction, suppose that $\mathcal{M}^{f}, \bar{s} \Vdash \diamond_{0} \psi$. Then, for some $\bar{t}$ in $\mathcal{M}^{f}$, we have that $R_{0} \bar{s} \bar{t}$ and $\mathcal{M}^{f}, \bar{t} \Vdash \psi$. Hence, by the inductive hypothesis, we have that $\mathcal{M}, t \Vdash \psi$. But then, from reflexivity of $\equiv_{0}$, it follows that $\mathcal{M}, t \Vdash \diamond_{0} \psi$, and so from $R_{0} \bar{s} \bar{t}$ we may infer, using only the definition of $R_{0}$, that $\mathcal{M}, s \Vdash \diamond_{0} \psi$.

This proves (7.8), so in order to prove the left-to-right direction of the proposition we have only to show that $\mathcal{M}^{f}$ is a pseudo-square. We leave it to the reader to verify that both $R_{0}$ and $R_{1}$ are equivalence relations. In order to check the other condition, let $\bar{s}$ and $\bar{t}$ be points in $\mathcal{M}^{f}$. Now the fact that $\mathcal{M}$ is a square and that $s$ and $t$ are pairs comes in handy. Let $z=\left(s_{0}, t_{1}\right)$. Then $s \equiv_{0} z \equiv_{1} t$. But it then follows that $R_{0} \bar{s} \bar{z}$ and $R_{1} \bar{z} \bar{t}$, which shows that the composition of $R_{0}$ and $R_{1}$ is indeed the universal relation on $\mathcal{M}^{f}$.

What can we conclude from the examples Tile, Tile ${ }^{-}$, and $\mathbf{S 5}^{\mathbf{2}}$ ? Not that looseness is a necessary condition for a modal system to be decidable: witness 
$\mathrm{Tile}^{-}$and $\mathbf{S} 5^{\mathbf{2}}$. On the other hand, it should be clear that dropping the looseness principle leads us to the immediate vicinity of the danger zone: adding only a grain of global expressive power will turn the highly decidable logic Tile $^{-}$into the undecidable Tile.

Concerning $\mathbf{S 5}^{\mathbf{2}}$, it is very interesting to observe what happens if we move to higher dimensions. For instance, there seem to be two three-dimensional counterparts of $\mathbf{S} 5^{\mathbf{2}}$, according to which relation between two triples one takes to be the accessibility relation for $\diamond_{i}$ :

$$
\begin{aligned}
& s \equiv_{i} t \text { if } s_{j}=t_{j} \text { for all } j \neq i, \\
& s \sim_{i} t \text { if } s_{i}=t_{i} .
\end{aligned}
$$

In the second, relatively loose, interpretation the resulting logic is decidable. In the first interpretation, one obtains a class of rather tight models; the resulting logic is undecidable. Since it is this logic that corresponds to a threevariable fragment of first-order logic (in a way similar to that discussed above for $\mathbf{S 5}^{\mathbf{2}}$ ), this makes an interesting case for the second locality principle.

\subsubsection{Universal access: $\mathrm{K}^{*}$}

We now consider the modal system $\mathbf{K}^{*}$ obtained by expanding the basic modal language with the modality $\langle *\rangle$, keeping the class of models intact and giving both $\diamond$ and $\langle *\rangle$ the standard interpretation. Recall that the meaning of $\langle *\rangle$ was defined using the reflexive transitive closure $R^{*}$ of the relation $R$.

Let us first see where $\mathbf{K}^{*}$ stands with respect to the looseness and locality principles. We have seen already that $\langle *\rangle$ is invariant under bisimulations, whence we have an analogue of Theorem 7.3.6: any $\mathbf{K}^{*}$-satisfiable formula is satisfiable in a tree model. $\mathbf{K}^{*}$ also meets the second locality principle, at least if we are allowed to include finite-variable fragments of the infinitary language $\mathcal{L}_{\omega_{1} \omega}$ (an extension of first-order logic in which countable conjunctions and disjunctions are allowed). For it is easy to see that $\mathbf{K}^{*}$-formulas have correspondents in the three-variable fragment of this language: simply add the following clause for $\langle *\rangle$ to the standard translation of $\mathcal{L}_{\diamond}$ :

$$
S T_{x}(\langle *\rangle \psi)=\exists y\left(R^{*} x y \wedge S T_{y}(\varphi)\right), \quad S T_{y}(\langle *\rangle \psi)=\exists x\left(R^{*} y x \wedge S T_{x}(\varphi)\right) .
$$

Here we use the fact that the reflexive transitive closure can be expressed using three variables only; for instance, $R^{*} x y$ could stand for the following abbreviation:

$$
x=y \vee R x y \vee \exists y^{\prime}\left(R x y^{\prime} \wedge R y^{\prime} y\right) \vee \exists y^{\prime}\left(\exists y\left(R x y \wedge R y y^{\prime}\right) \wedge R y^{\prime} y\right) \vee \ldots
$$

However, $\mathbf{K}^{*}$ violates the first locality principle in the following way: if $r$ is the root of a tree model $\mathcal{M}$, then we have

$$
\mathcal{M}, r \Vdash\langle *\rangle \varphi \leftrightarrow \mathcal{M}, s \Vdash \varphi \text { for some } s \text { in } \mathcal{M}
$$


as the reader can easily check. In fact, unlike the basic modal system, $\mathbf{K}^{*}$ does not have the finite tree model property; for instance, the following satisfiable formula is not satisfiable on any finite tree:

$$
[*](p \rightarrow\langle *\rangle \neg p) \wedge[*](\neg p \rightarrow\langle *\rangle p) .
$$

(It is satisfiable on the natural numbers with successor, with $p$ interpreted as the even numbers.)

Summarizing, the present system is loose, and it satisfies the second but not the first locality principle. What about its decidability, or the finite model property? In fact, both properties hold, as we shall see now. We first prove that $\mathbf{K}^{*}$ has the bounded model property.

Proposition 7.4.4. Any satisfiable $\mathbf{K}^{*}$ formula $\xi$ is satisfiable on a model of size $2^{O(|\xi|)}$.

Proof. Suppose that $\xi$ is satisfiable in some model $\mathcal{M}=(W, R, V)$. We again define a collection of relevant formulas. This time, we need a new closure rule: we call a set $X$ of formulas $*$-closed if it contains $\diamond\langle *\rangle \varphi$ whenever it contains $\langle *\rangle \varphi$. Now let $F L(\xi)$ be the smallest set of formulas containing $\xi$ which is *-closed, besides being closed under taking subformulas and single negations. It is not difficult to prove that the cardinality of $F L(\xi)$ is linear in the size of $\xi$.

The method that we use to construct a finite model for $\xi$ is, just as in the case of $\mathbf{S 5}^{\mathbf{2}}$, that of filtration. We define the following relation on points of $\mathcal{M}$ :

$$
s \sim s^{\prime} \leftrightarrow \text { for all } \varphi \text { in } F L(\xi): \mathcal{M}, s \Vdash \varphi \text { iff } \mathcal{M}, s^{\prime} \Vdash \varphi .
$$

Again, it is obvious that $\sim$ is an equivalence relation, and again, our filtrated model will be based on the collection $W^{f}$ of equivalence classes of this relation; it is convenient to identify the equivalence class of $s$ with the color of $s$, which we define as the set $c(s)=\{\varphi \in F L(\xi) \mid \mathcal{M}, s \Vdash \varphi\}$. Note that $\left|W^{f}\right| \leq 2^{O(|\xi|)}$, as $|F L(\xi)|$ is bounded by $|\xi|$.

To finish the definition of the filtrated model, we define the relation $R^{f}$ on colors as follows:

$$
R^{f} c d \leftrightarrow \text { for all } \diamond \varphi \in F L(\xi):(\varphi \in d \Rightarrow \diamond \varphi \in c) .
$$

The valuation $V^{f}$ is then defined as $V^{f}(p)=\left\{c \in W^{f} \mid p \in c\right\}$.

The key claim of the filtration proof is the following.

Claim 1. For all formulas $\varphi \in F L(\xi)$ and all colors $c, \varphi \in c$ iff $\mathcal{M}^{f} \Vdash \varphi$.

Proof of Claim The proof follows by an induction on the complexity of $\varphi$. We treat only the case where $\varphi$ is of the form $\langle *\rangle \psi$.

First suppose that $\langle *\rangle \psi \in c$. Assume that $c$ is the color of $s$ in $\mathcal{M}$; that is, $\mathcal{M}, s \Vdash\langle *\rangle \psi$. By definition, there is a sequence of states $s_{1}, \ldots, s_{n}$ in $\mathcal{M}$ such that $s=s_{1}, R s_{i} s_{i+1}$ for all $i$, and $\mathcal{M}, s_{n} \Vdash \psi$. By the definition of colors, it 
follows that $\psi \in c\left(s_{n}\right)$. Also, it is easy to show that $R^{f} c\left(s_{i}\right) c\left(s_{i+1}\right)$ for all $i$. But then it follows immediately that $\mathcal{M}^{f}, c \Vdash\langle *\rangle \psi$.

For the other direction, suppose that $\mathcal{M}^{f}, c \Vdash\langle *\rangle \psi$. By the truth definition of $\langle *\rangle$, there must be colors $c_{1}, \ldots, c_{n}$ such that $c=c_{1}, R^{f} c_{i} c_{i+1}$ for all $i$, and $\mathcal{M}, c_{n} \Vdash \psi$. It follows from the inductive hypothesis that $\psi \in c_{n}$. From this, and the observation that $\varphi \rightarrow\langle *\rangle \varphi$ is valid in any model, it follows that $\langle *\rangle \psi \in c_{n}$.

We now show that

$$
\text { If } R^{f} d d^{\prime} \text {, then }\langle *\rangle \chi \in d^{\prime} \text { implies }\langle *\rangle \chi \in d \text {. }
$$

Suppose that $R^{f} d d^{\prime}$ and $\langle *\rangle \chi \in d^{\prime}$. It follows that $\langle *\rangle \chi$ belongs to $F L(\xi)$, and so $\diamond\langle *\rangle \chi$ is in $F L(\xi)$ as well, by $*$-closure. But then, by the definition of $R^{f}$, we find that $\diamond\langle *\rangle \chi$ is in $d$. Since $d$ is a color, there must be some $w$ in $W$ such that $d=c(w)$. By definition, we have that $\mathcal{M}, w \Vdash \diamond\langle *\rangle \chi$. From this it is easy to derive that $\mathcal{M}, w \Vdash\langle *\rangle \chi$, and so again, by definition, we have $\langle *\rangle \chi \in d$.

But, from (7.9) and $\langle *\rangle \psi \in c_{n}$, an easy downward inductive proof shows that $\langle *\rangle \psi \in c_{i}$ for all $i$. In particular, we find that $\langle *\rangle \psi$ belongs to $c_{1}=c$. This finishes the proof of the claim.

Thus $\xi$ is satisfiable in a model of size $2^{O(|\xi|)}$.

The last proposition implies decidability, as it is decidable whether a $\mathbf{K}^{*}$ formula is satisfiable on a finite model. The idea of colors can also be used directly in an algorithm which tries to construct a model like $\mathcal{M}^{f}$. This construction uses the same idea as the $K$-World algorithm given earlier: states are identified with subsets of $C l(\xi)$. Let $S_{0}$ consists of all sets $\Delta \subseteq C l(\xi)$ for which $\operatorname{Prop}-\operatorname{Max}(\Delta, C l(\xi))$ holds and which satisfy $\varphi \in \Delta \Rightarrow\langle *\rangle \varphi \in \Delta$, for all $\langle *\rangle \varphi \in C l(\xi)$. (For the definition of $\operatorname{Prop}-\operatorname{Max}(\Delta, C l(\xi)$ ), see section 7.3.) Clearly $S_{0}$ can be effectively computed and $\left|S_{0}\right| \leq 2^{O(|\xi|)}$. We now inductively construct a sequence of collections of sets of formulas $S_{0} \supsetneq S_{1} \supsetneq S_{2} \supsetneq S_{3} \cdots$. During this construction, just as in the K-World algorithm, we try to find witnesses for diamond formulas. We say that a set $\Delta \in S_{i}$ is ready if $S_{i}$ contains witnesses for all diamond formulas in $\Delta$ :

- for every formula $\diamond \psi \in \Delta$ there is a $\Delta_{\psi} \in S_{i}$ such that $R^{f} \Delta \Delta_{\psi}$ and $\psi \in \Delta_{\psi}$, and

- for every formula $\langle *\rangle \psi \in \Delta$ there are $\Delta_{1}, \Delta_{2}, \ldots, \Delta_{n} \in S_{i}$ such that $\Delta=$ $\Delta_{1}, R^{f} \Delta_{i} \Delta_{i+1}$ and $\psi \in \Delta_{n}$,

If every set in $S_{i}$ is ready and $S_{i}$ contains a set $\Delta$ with $\xi \in \Delta$, then the algorithms returns " $\xi$ is satisfiable". If there is no set in $S_{i}$ containing $\xi$, then the algorithms returns " $\xi$ is not satisfiable". Otherwise, let $S_{i+1}$ consist of all ready sets in $S_{i}$, and we continue the construction. Since $S_{i} \supsetneq S_{i+1}$, the construction is guaranteed to terminate in at most $2^{O(|\xi|)}$ stages. The correctness of the algorithm can be shown along the lines of the proof of the last proposition. Thus we have established the following.

Theorem 7.4.5. It is decidable whether a given $\mathbf{K}^{*}$ formula is satisfiable. 


\subsubsection{Generalizing looseness: the until operator}

In this subsection, we consider the modal system given by the propositional language expanded with the binary until operator $U$, the class of all models of the form $(W, R, V)$, and an interpretation of $U$ as given above (recalled below). We have already mentioned that truth in this language is not bisimulationinvariant, and we are thus not dealing with a modal system in the narrow sense; in particular, we shall see that there are satisfiable $U$-formulas that are not satisfiable in any tree. Nevertheless, we shall show that this system does have some kind of loose model property, and we shall use this property for showing that it has a decidable satisfiability problem. In fact, this "looseness property" is the reason why we take a look at this operator: it shows in a relatively simple setting how to generalize the notions of looseness and tree models. These generalizations are made in the section on guarded fragments.

To start with, let $\mathcal{L}_{U}$ be the language obtained by expanding the classical propositional language with the binary connective $U$. Recall that $\mathrm{M}$ is the class of all models of the form $(W, R, V)$. It is convenient to use the following notation: for $s$ and $u$ elements of $W$,

$$
\mathcal{M}, s u \Vdash \psi \text { iff } \mathcal{M}, t \Vdash \psi \text {, for all } t \text { satisfying } R s t \text { and } R t u \text {. }
$$

This is because we can now rephrase the truth definition of the until operator as follows:

$$
\mathcal{M}, s \Vdash U(\varphi, \psi) \text { iff } \mathcal{M}, u \Vdash \varphi \text { and } \mathcal{M}, s u \Vdash \psi \text {, for some } u \text { such that } R s u \text {. }
$$

We call the resulting modal system $\left(\mathcal{L}_{U}, \mathrm{M}, \Vdash\right)$ the until system. In order to see why truth of $\mathcal{L}_{U}$-formulas is not invariant under bisimulations, consider the formula $U(p, \top) \wedge \neg U(p, p)$. (Here $\top$ abbreviates $(p \vee \neg p)$.) This formula is satisfiable and its smallest irreflexive model contains three points; see model $\mathcal{M}_{1}$ in Figure 7.7. Note that in the unraveling $\mathcal{M}_{2}$ of the model $\mathcal{M}_{1}, U(p, p)$ holds at the root of the tree. This shows that $\mathcal{L}_{U}$ is really a more expressive language than $\mathcal{L}_{\diamond}$. In fact, one can show that the formula $U(p, \top) \rightarrow U(p, p)$ holds throughout any tree model, whence $U(p, \top) \wedge \neg U(p, p)$ is not satisfiable in any tree model. This shows that the until system does not have the tree model property.

\section{Decidability}

Unlike our earlier proofs, we shall not use any kind of finite model property in order to prove decidability for the until system. This is not because the system does not have the bounded finite model property (it does); our proof method is for didactic purposes. The idea behind the mosaic method that we employ is that instead of transforming a model into a finite model, we could just as well "deconstruct" it into a finite "toolkit", which we shall call a linked 


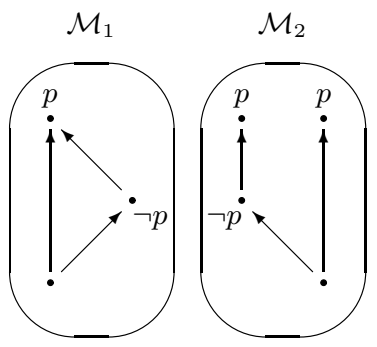

Fig. 7.7. Until formulas are not invariant under bisimulations

set of mosaics. One then has to show that a formula is satisfiable if and only if there exists such a linked set of mosaics for it.

What, then, are mosaics? One could best describe them as little pieces of a model that, if linked together in a nice way, contain sufficient information to reconstruct another model which looks sufficiently like the original one to preserve the truth of $\mathcal{L}_{U}$-formulas. In this way we will establish a loose model property for the until system: any satisfiable formula is satisfiable in a model consisting of these isomorphic copies of mosaics that hang together only very loosely. (Later on in the chapter, we shall come back to this issue in more technical detail.)

Concerning the notion of a mosaic, the first question is what information we are interested in. This question is easy to answer: as in the filtration proof for $\mathbf{S} 5^{\mathbf{2}}$, we are interested only in the truth of subformulas of $\xi$. The second question then should be: which parts are we going to cut out of the model? Here we need to define a new concept. We call a subset of the domain of a model $\mathcal{M}=(W, R, V)$ packed if every two distinct elements $s$ and $t$ of the subset are $R$-related (that is, we require that $R s t$ or $R t s$ ). Our patchwork pieces will then be packed sets of size at most three.

The number three here derives from the fact that the truth definition of $U(\varphi, \psi)$ employs three variables. In fact, if one were to try to devise a standard translation or a bisimulation game for the $\mathcal{L}_{U}$-language, the number three would show up as the minimal number of variables needed and as the minimal size of the windows that cover the models during the game. During a game, one would see that these windows would be placed only on packed sets of the models.

Abstracting from the origin of these pieces, we arrive at the following definition. From now on, we let $\xi$ be an arbitrary but fixed $\mathcal{L}_{U}$ formula; $\xi$ is the formula whose satisfiability needs to be decided. We let $C l(\xi)$ denote the set of subformulas of $\xi$.

Definition 7.4.6. $A$-type mosaic is a quadruple $\mu=\left(X, R, A_{\varphi}, B_{\varphi}\right)_{\varphi \in C l(\xi)}$ such that $X$ is a set of size at most three; $R$ and every $B_{\varphi}$ are binary relations 
on $X$; and every $A_{\varphi}$ is a unary relation on $X$. When $\xi$ is clear from the context, we shall use simply the term "mosaic".

The basic idea underlying this definition is that $A_{\varphi}$ holds of a point if we "want" $\varphi$ to be true at it, while $B_{\varphi}$ holds of a pair of points if we "want" $\varphi$ to be true at every point between them. Obviously, not every such structure is part of a model - we need some further constraints for that. We call a mosaic coherent if it satisfies the following conditions (phrased in first-order logic and to be read universally):

(C0) $R x y \vee R y x \vee x=y$,

(C1) $A_{\neg \varphi} x \leftrightarrow \neg A_{\varphi} x$,

(C2) $A_{\varphi \wedge \psi} x \leftrightarrow A_{\varphi} x \wedge A_{\psi} x$,

(C3) $B_{\varphi \wedge \psi} x y \leftrightarrow B_{\varphi} x y \wedge B_{\psi} x y$

(C4) $\left(R x y \wedge R y z \wedge B_{\varphi} x z\right) \rightarrow A_{\varphi} y$

$(C 5)\left(R x y \wedge A_{\varphi} y \wedge B_{\psi} x y\right) \rightarrow A_{U(\varphi, \psi)} x$.

A few words of explanation: $\mathrm{C} 0$ reflects the fact that we have taken only packed subsets of the model as the domain of our mosaic mini-models. C1-C3 are selfexplanatory; note that there is no analog of $\mathrm{C} 1$ for the $B$-predicates, since there is a hidden universal quantifier in the meaning of a predicate $B_{\varphi}$, see (7.10). Finally, C4 and C5 are rather obvious consequences of our intuitive meaning of the $A$ - and $B$-predicates and the truth definition of the until operator.

The conditions $\mathrm{C} 0-\mathrm{C} 5$ take care of all universal constraints on the $A$ - and $B$-predicates; but of course there are existential demands as well, which we shall call requirements. A requirement of a mosaic $\mu=\left(X, R, A_{\varphi}, B_{\varphi}\right)_{\varphi \in C l(\xi)}$ is one of the two following types of object:

(a) $\left(A_{U(\varphi, \psi)}, s\right)$ such that $A_{U(\varphi, \psi)} s$,

(b) $\left(\right.$ not $\left.B_{\varphi}, s, t\right)$ such that $R s t$ and not $B_{\varphi} s t$.

In order to explain the requirements of type (a), suppose that we want the formula $U(\varphi, \psi)$ to be true at a point $s$; if there is a point $t$ in the mosaic such that Rst, $A_{\varphi} t$, and $B_{\psi} s t$, then the mosaic itself directly fulfills the requirement. This will rarely be the case, however; the whole point of the mosaic method is that requirements can be fulfilled by distinct mosaics as well, as follows. A link between two mosaics $\mu$ and $\mu^{\prime}$ is simply a partial isomorphism between the two structures. We say that a link $f: \mu \hookrightarrow \mu^{\prime}$ fulfills the requirement $\left(A_{U(\varphi, \psi)}, s\right)$ of $\mu$ if there is some $t$ in $\mu^{\prime}$ with $R f(s) t, A_{\varphi} t$, and $B_{\psi} f(s) t$. Likewise, a link $f: \mu \hookrightarrow \mu^{\prime}$ fulfills the requirement $\left(\operatorname{not} B_{\varphi}, s, t\right)$ if there is some $u$ in $\mu^{\prime}$ with $R f(s) u, R u f(t)$, and $\neg A_{\varphi} u$.

A collection $L$ of mosaics is called a linked set of mosaics if every requirement of every mosaic $\mu \in L$ is fulfilled via some link $f: \mu \hookrightarrow \mu^{\prime}$ to some $\mu^{\prime}$ also in $L$. It is a linked set of mosaics for $\xi$ if it contains a mosaic with nonempty $A_{\xi}$.

The main theorem concerning mosaics is the following. (In order to follow the main line of the chapter, the reader could skip the details of the proof.) 
Proposition 7.4.7. An $\mathcal{L}_{U}$-formula $\xi$ is satisfiable if and only if there is a linked set of mosaics for $\xi$.

Proof. The left-to-right direction of the proof is easy. Suppose that $\mathcal{M}=$ $(W, R, V)$ is a model for $\xi$. Out of this model, we cut a linked set of mosaics for $\xi$, as follows. Let $X$ be the collection of triples $\vec{x}=\left\langle x_{1}, x_{2}, x_{3}\right\rangle$ such that $R x_{1} x_{2}, R x_{2} x_{3}$, and $R x_{1} x_{3}$. Associate with any such triple a mosaic $\mu_{\vec{x}}$ based on the set $\left\{x_{1}, x_{2}, x_{3}\right\}$, with $R$ as in $\mathcal{M}$ and with every $A_{\varphi}$ and $B_{\varphi}$ defined as given by the truth of $\varphi$ in $\mathcal{M}$. We leave it as an exercise for the reader to verify that the collection of all these mosaics indeed forms a linked set of mosaics.

The right-to-left direction of the proposition is the hard one, although the key idea underlying its proof is quite intuitive. We construct a model for $\xi$ step by step; that is, we approximate our model via a series of finite structures that we call networks. A network is a structure $\mathcal{N}=\left(W, R, A_{\varphi}, B_{\varphi}\right)_{\varphi \in C l(\xi)}$ of the same type as a mosaic but not bounded in size. A network is called coherent if it satisfies the conditions $\mathrm{C} 1-\mathrm{C} 5$ above. To ask for $\mathrm{C} 0$ would be too much; instead, we require coherent networks to satisfy the following:

(liveness) every packed set $X$ of size at most three comes from a mosaic; that is, for each such set $X \subseteq W$ there is a partial isomorphism $f: \mathcal{N} \hookrightarrow \mu$ such that $f$ is defined on $X$.

Liveness means that, through the mosaics, we are in control of certain small parts of the model: the packed sets of size at most three. Why only these sets? The truth definition of $U$ provides the answer. The meaning of $U(\varphi, \psi)$ depends only on these small packed sets in the model.

A defect of a network is a requirement that is not directly fulfilled in the network itself, and a network is called saturated if it has no defects. A network is perfect if it both coherent and saturated.

This name is well chosen, since perfect networks are the ones that we are after. The reason for this is that with every network $\mathcal{N}=\left(W, R, A_{\varphi}, B_{\varphi}\right)_{\varphi \in C l(\xi)}$ we can associate a modal model in an obvious way: it is defined as the structure $\mathcal{N}^{\circ}=\left(W, R, V^{\circ}\right)$, where $V^{\circ}(p)=A_{p}$ for all variables $p$ occurring in $\xi$. But only for perfect networks can we prove the following truth lemma.

Claim 1. If $\mathcal{N}$ is a perfect network, then for all formulas $\varphi \in C l(\xi)$ and all points $s, t$ in $\mathcal{N}$ :

1. $s \in A_{\varphi}$ iff $\mathcal{M}, s \Vdash \varphi$.

2. If $R s t$, then $(s, t) \in B_{\varphi}$ iff $\mathcal{M}, s t \Vdash \varphi$.

Proof of Claim The proof of this claim is by induction on the complexity of $\varphi$. We consider only the case where $\varphi$ is of the form $U(\psi, \chi)$, and only prove part 2 of the claim (the first part is simpler).

By the induction hypothesis and the truth definition of $U$, in order to prove part 2 it suffices to show that for all pairs of points $s$ and $t$ such that $R s t$, we have that $(s, t) \notin B_{\varphi}$ iff $u \notin A_{\varphi}$ for some $u$ with $R s u$ and $R u t$. 
The left-to-right direction immediately follows from the fact that $\mathcal{N}$ is perfect and thus all requirements of type (b) are fulfilled. For the other direction, suppose that $s, t$ and $u$ are points satisfying $R s t, R s u, R t u$ and $u \notin A_{\varphi}$. Observe that $\{s, t, u\}$ is a packed set of size at most three, so that we may use the liveness condition. This yields a partial isomorphism $f$ from $\mathcal{N}$ to some mosaic $\mu$ such that $f$ is defined for each of $s, t$, and $u$. It follows that $R f(s) f(t)$, $R f(s) f(u), R f(t) f(u)$, and $f(u) \notin A_{\varphi}$; but then it follows from condition C4 that $(f(s), f(t)) \notin B_{\varphi}$. Returning to $\mathcal{N}$, this shows that $(s, t) \notin B_{\varphi}$, which is what we needed to prove. This finishes the proof of the claim.

It follows from the above claim that in order to show that $\xi$ is satisfiable, it suffices to show that there is a perfect network for it, that is, a perfect network such that $A_{\xi}$ is not empty.

Claim 2. There is a perfect network for $\xi$.

Proof of Claim The proof of this claim falls out into three parts. First we show that there is some network for $\xi$ (not necessarily perfect). This is easy, since we are given a linked set of mosaics for $\xi$ : as our network we simply take any mosaic with a nonempty $A_{\xi}$.

The second and main part of the proof consists in showing that any defect of any network can be repaired; that is, we can find a bigger network in which the defect no longer occurs. Without going into too much technical detail, let us see how to repair a defect of type (b) (defects of type (a) are repaired in a similar way).

Suppose that $s$ and $t$ are points of the network $\mathcal{N}$ such that $R s t$ and not $B_{\varphi} s t$ for some subformula $\varphi$ of $\xi$, while there is no point $u$ between $s$ and $t$ such that $\neg A_{\varphi} u$. The idea now is simply to repair this defect by adding a new point to the network. What kind of point? Well, since we have Rst we know that $s$ and $t$ come from a mosaic; that is, there is a partial isomorphism $f$ from $\mathcal{N}$ to some mosaic $\mu$. Obviously, $\left(\operatorname{not} B_{\varphi}, f(s), f(t)\right)$ is a requirement of this mosaic. But since we are working with a linked set of mosaics, there must be some link $g$ between $\mu$ and $\mu^{\prime}$ and some $u$ in $\mu^{\prime}$ such that $R g(f(s)) u$, $R u g(f(t))$, and $\neg A_{\varphi} u$. Now we simply add an entirely new point $r$ to the network, and make sure that the relations between $s, t$, and $r$ are such that this part of the model is isomorphic to $\mu^{\prime}$. It is thus obvious that we have repaired the defect, and that the new structure is a network. In order to keep the liveness condition, it is essential not to relate $r$ to any other point besides $s$ and $t$ : in this way, the only new packed sets are $\{r, s, t\}$ and its subsets.

Finally, these two parts provide the material and the tools for constructing the desired perfect network for $\xi$. Starting from the mosaic for $\xi$ (which is of course a network), we repair defects, one by one, step by step, thus constructing a sequence $\mathcal{N}_{0}, \mathcal{N}_{1}, \ldots$ of networks. Using some standard combinatorics, we can ensure that the limit of the chain of networks is a network without defects. In particular, if we always take new points from a fixed set, say $\omega$, we can enumerate the set of all (potential) defects of any network in the chain; 
if at each step of the construction we repair the current network's defect with the lowest number in this enumeration, we can create a perfect network. This finishes the proof of the claim.

Theorem 7.4.8. It is decidable whether a given $\mathcal{L}_{U}$-formula is satisfiable.

Proof. We can adjust the "elimination algorithm" given for the system $\mathbf{K}^{*}$ in order to deal with mosaics. This is done as follows. Let $S$ be the set of all $\xi$-type mosaics (up to isomorphism). Let $S_{0} \subseteq S$ be the subset containing all coherent mosaics. $S_{0}$ can be computed effectively, since coherence can be checked effectively. It is not hard to show that $|S| \leq 2^{O(|\xi|)}$. We now inductively construct a sequence of sets of mosaics $S_{0} \supsetneq S_{1} \supsetneq S_{2} \supsetneq S_{3} \ldots$, just as in the proof for the system $\mathbf{K}^{*}$. The idea is that we delete mosaics from $S_{i}$ if they have a requirement which cannot be fulfilled inside $S_{i}$. The details of this construction will be spelled out in the section on guarded fragments.

What is important to remember is that the until system has a kind of loose model property: if a formula $\xi$ is satisfiable then there is a linked set of mosaics for it, and if there is such a set for $\xi$, then the proof of Proposition 7.4.7 shows how to construct a loose model for $\xi$. We shall come back to this in Section 7.6

\section{Notes}

Tiling problems (or domino problems, as they are sometimes called) were introduced by Wang [61] and have since been used in a variety of forms to prove undecidability and complexity results. An accessible proof of the undecidability of the $\mathbb{N} \times \mathbb{N}$ tiling problem, a result due to Berger [8], can be found in the monograph by Börger, Grädel \& Gurevich [10]. Our discussion of the logic Tile was based on Spaan [55], where an example is presented in a language that expands the basic modal language with the universal diamond.

The modal system $\mathbf{S 5}^{\mathbf{2}}$ (S5 square) has a long history in the algebraic disguise of the class of diagonal-free cylindric algebras of dimension two, see the monograph [27]. The bounded finite model property of $\mathbf{S 5}^{2}$ was first established by Segerberg [53]. The fact that every pseudo-square bisimulates by a functional bisimulation with a square can be found in Marx \& Venema [43]. The higher-dimensional counterparts of $\mathbf{S} 5^{\mathbf{2}}$ are studied as modal logics in Venema [59,60].

The modal system $\mathbf{K}^{*}$ can best be seen as a fragment of propositional dynamic logic (PDL) in which there is only one atomic program. For more information on PDL, the reader is referred to the handbook article by Harel [24]. The decidability of PDL was proved by Fischer \& Ladner [18]. The elimination algorithm leading to Theorem 7.4.5 is due to Pratt [50].

The operators "Since" and "Until" were introduced by Hans Kamp in order to prove expressive (in)completeness results for temporal logics over classes of linear flows of time. Nowadays, they belong to the standard repertoire of 
temporal logics in computer science; see [39]. A bisimulation variant which characterizes this language over arbitrary models was found by Kurtonina \& de Rijke [36]; for some decidability results over classes of linear flows of time, see Burgess \& Gurevich [12]. Our Theorem 7.4.8 and its proof are based on results and proofs related to the loosely guarded fragment that are due to van Benthem.

The filtration method has been used extensively as a tool for proving decidability results for modal logics, since Lemmon [38] and Segerberg [52] further developed ideas dating back to McKinsey \& Tarski [44]. The mosaic method for proving decidability of a logic was developed by Németi [45]; it has since been used for a wide range of logics, often related to a multi-dimensional modal setting. With hindsight, even Gödel's proof of the decidability of the satisfiability problem for $\forall^{2} \exists^{*}$ prenex sentences can be called a mosaic-style proof as well; see the very clear exposition in [10].

\subsection{Modal Complexity}

In the previous sections, we have discussed the decidability of the satisfiability problem for several modal systems, gathering various results along the way. For instance, for the basic modal system $\mathbf{K}$, we saw that every satisfiable formula can be satisfied in an exponential-size tree model with branches of polynomial depth; for $\mathbf{S 5}^{2}$, we could do no better than finding an exponentialsize quasi-model.

In this section, we take a closer look at such differences, examining how they affect the complexity of the modal systems that we present. Our goal is not to give precise reductions and matching algorithms - this is very well documented in the literature. Rather, we shall paint with a broad brush and try to convey once more our earlier message that looseness and locality are key notions in understanding the decidability and complexity of modal systems. To do this we discuss modal systems whose satisfiability problems are complete for the complexity classes NP, PSPACE, EXPTIME, and NEXPTIME, respectively. We believe that these systems, besides being complete for these classes, indeed form very indicative examples.

Our agenda for the section is set out in Table 7.1. We assume that the reader has at least a basic understanding of complexity classes such as NP, PSPACE, EXPTIME, etc. Completeness and hardness are understood in this chapter by means of polynomial-time many-one reductions. (The reader is referred to [3] for basic definitions.) In this section, we concentrate on the satisfiability problem - recall that C-completeness of the satisfiability problem for a modal system implies co-C-completeness of the validity problem.

The layout of this section is summarized in Table 7.1. Every column represents a modal system and the complexity class for which its satisfiability problem is complete. The third row indicates whether satisfiable formulas can be satisfied 
in tree models for that logic, and the fourth row whether the modal system is expressive enough to define the universal modality. We mention these two properties because they correspond to the looseness and first locality principle, respectively (as mentioned before, we shall meet the second locality principle again in the last section). The star in the first column marks the special role of the logic S5, which is the logic of the universal modality $\mathrm{E}$ by itself.

\begin{tabular}{|l||c|c|c|c|c|}
\hline modal system/logic & S5 & Func & K & $\mathbf{K}^{*}$ & $\mathbf{S 5}^{\mathbf{2}}$ \\
\hline complete for & NP & NP & PSPACE & EXPTIME & NEXPTIME \\
\hline tree model property & $*$ & yes & yes & yes & no \\
\hline global expressive power & yes & no & no & yes & yes \\
\hline subsection & 7.5 .1 & 7.5 .1 & 7.5 .2 & 7.5 .3 & 7.5 .4 \\
\hline
\end{tabular}

Table 7.1. Layout of Section 7.3.

We shall often use the tractability result concerning model checking that we mentioned earlier on when discussing the second locality principle. In this section, we confine ourselves to modal languages with a finite number of firstorder definable modal operators. For modal formulas in such a language, the model checking problem (i.e., given as input a (finite) model $\mathcal{M}$, a state $s$ and a formula $\xi$, to determine whether $\mathcal{M}, s \Vdash \xi$ ), is solvable in PTIMEin the size of both the model and the formula. Also, for an elementary (i.e., definable by a single first-order sentence) class of models $\mathrm{K}$, the membership problem (given as input a finite model $\mathcal{M}$, to determine whether $\mathcal{M}$ belongs to $\mathrm{K}$ ), is also solvable in PTIME. We shall call a modal system elementary if it has an elementary class of models and each of its operators has a first-order truth definition.

\subsubsection{NP and the polysize model property}

The class NP of nondeterministic polynomial-time algorithms is the smallest complexity class that we shall consider for the satisfiability problem for modal systems. The reason for this is that every nontrivial modal logic contains the collection of all valid propositional formulas; hence we can reduce the NPcomplete satisfiability problem for propositional logic to that of the modal system. So NP is a nice class to work with since we only have to show an upper bound. Unfortunately, there are not many modal systems with a satisfiability problem in NP.

How can we show that the satisfiability problem is in NP for a given modal system? The easiest route and the one that modal logicians most often take is via the polysize model property. A modal system is said to have this property if every satisfiable formula $\xi$ is satisfiable in a model whose size is bounded by $p(|\xi|)$ for a fixed polynomial $p$. Using the two complexity results mentioned 
above, namely, PTIME for both the model checking and the membership problem, it is easy to show that for elementary modal systems, the polysize model property implies NP-completeness of the satisfiability problem.

Let us see then, if we can find modal systems with this polysize model property; we shall confine ourselves to the basic modal language. In section 7.3, we showed that every satisfiable formula $\varphi$ can be satisfied in an at most $|\varphi|$ ary tree of depth at most $|\varphi|$ : a model whose size is exponential in $|\varphi|$. Thus, if we want a polysize model, we should restrict either the width or the depth of such trees. This is possible if we consider smaller classes of models.

Restricting the width is easy: we consider only models in which $R$ is a total function. The cut-off argument in Section 7.3 yields a linear-sized model. Recall from Definition 7.3.8 that $d_{\diamond}(\xi)$ denotes the modal depth of $\xi$.

Proposition 7.5.1. Let Func be the modal system $\left(\mathcal{L}_{\diamond}, \Vdash, \mathrm{F}\right)$ such that $\Vdash$ is the standard definition and $\mathrm{F}$ is the class of models in which $R$ is a total function. Let $\xi$ be a formula in $\mathcal{L}_{\diamond}$. If $\xi$ is Func-satisfiable, then it is Funcsatisfiable in a model containing at most $d_{\diamond}(\xi)+1$ states.

As a corollary, the satisfiability problem for Func is in NP.

An extremely simple way of bounding the depth is to make the accessibility relation total; a state at which $\varphi$ holds is then a witness for $\diamond \varphi$ at every state in the model.

Note, however, that making the accessibility relation total breaks with the first locality principle! Nevertheless, the resulting modal system, which we shall call S5 after the name of the logic associated with it, has a satisfiability problem in NP.

Proposition 7.5.2. Let $\mathbf{S 5}$ be the modal system $\left(\mathcal{L}_{\diamond,} \Vdash, \mathrm{U}\right)$ such that $\Vdash$ is the standard definition and $\mathrm{U}$ is the class of models in which $R$ is the universal relation. Let $\xi$ be a formula in the basic modal language. If $\xi$ is $\boldsymbol{S} 5$-satisfiable then it is $\boldsymbol{S 5}$-satisfiable in a model containing at most $|\xi|$ states.

As a corollary, the satisfiability problem for $\boldsymbol{S} \boldsymbol{5}$ is in NP.

Proof. Let $\mathcal{M}=(M, R, V)$ be a model such that $R=M \times M$ and $\mathcal{M}, s \Vdash \xi$. Choose, for every subformula $\diamond \varphi$ of $\xi$, a state $t \in M$ such that $\varphi$ holds at $t$ (if such a state exists). Let $\mathcal{M}^{\prime}$ be the submodel of $\mathcal{M}$ consisting of $s$ plus the selected states. By our pruning argument of Section 7.3, $\mathcal{M}^{\prime}, s \Vdash \xi$, because $R$ is the universal relation.

This finishes our discussion of NP and the polysize model property. Modal systems with this property are few and far between. In the next subsection, we shall see that for the basic modal system we can get only an exponential upper bound on the size of a model.

\subsubsection{PSPACE and polynomially deep paths}

In Section 7.3 we showed that every satisfiable formula $\xi$ in the basic modal language is satisfiable in a finite tree model $\mathcal{M}$, with depth and branching 
degree both bounded by the length $|\xi|$ of the formula. The good news about this argument is that it can be used to show that satisfiability for $\mathbf{K}$ can be decided in PSPACE. On the other hand, the upper bound that it establishes on the size of $\mathcal{M}$ is no better than exponential - at this stage, the reader might wonder whether this is an optimal bound. Here we show that it is up to a polynomial. In fact, the satisfiability problem of $\mathbf{K}$ is complete for PSPACE.

We now define, for each natural number $n$, a satisfiable formula $\xi(n)$ with the following two properties:

- the size of $\xi(n)$ is quadratic in $n$; and

- when $\xi(n)$ is satisfied in any model $\mathcal{M}$ at state $s$, then $\mathcal{M}$ contains as a substructure an isomorphic copy of the binary tree of depth $n$ whose root is $s$.

Thus the size of the smallest model satisfying $\xi(n)$ is exponential in $|\xi(n)|$. The idea underlying the definition of $\xi(n)$ is very simple: take $n$ propositional variables $p_{0}, \ldots, p_{n-1}$, and write a formula which, when satisfied, forces a binary-branching tree in which every possible valuation on $\left\{p_{0}, \ldots, p_{n-1}\right\}$ occurs at some leaf. Thus the model certainly contains $2^{n}$ different states. The formula is constructed using two "macros": $\operatorname{branch}\left(p_{i}\right)$ and store $\left(p_{i}\right)$ defined as follows:

$$
\begin{aligned}
& \operatorname{branch}\left(p_{i}\right):=\diamond\left(p_{i} \wedge \square p_{i}\right) \wedge \diamond\left(\neg p_{i} \wedge \square \neg p_{i}\right) \\
& \operatorname{store}\left(p_{i}\right):=\left(p_{i} \rightarrow \square p_{i}\right) \wedge\left(\neg p_{i} \rightarrow \square \neg p_{i}\right) .
\end{aligned}
$$

The formula $\xi(n)$ is then given by

$$
\operatorname{branch}\left(p_{0}\right) \wedge \bigwedge_{1 \leq i<n} \square^{i}\left(\operatorname{branch}\left(p_{i}\right) \wedge \bigwedge_{0 \leq j<i} \operatorname{store}\left(p_{j}\right)\right),
$$

in which $\square^{i}$ abbreviates a sequence of boxes, of length $i$. The formula works as follows. Suppose $\mathcal{M}, s \Vdash \xi(n)$. Then the branch part of $\xi(n)$ states that every node $t$ reachable in $i R$-steps from $s$ has two different successors, one forcing $p_{i} \wedge \square p_{i}$ and the other forcing $\neg p_{i} \wedge \square \neg p_{i}$. The store part of the formula states that successors of $t$ created by the branch part satisfy precisely the same proposition letters $p_{0}, \ldots, p_{i-1}$ as does $t$. We leave it to the reader to verify that the interplay of the branch and store macros forces a binary tree of depth $n$, as desired.

PSPACE lower bound. Of course, failure of the polysize model property for the basic modal system does not in itself imply that its satisfiability problem cannot be decided in NP. However, in fact the lower bound of this problem is known to be PSPACE. This result can be obtained by an interpretation of the validity problem of quantified boolean formulas. This interpretation is based on the same two macros branch and store.

In a similar way, one can establish the existence of exponential-sized models and a PSPACE lower bound for the modal system with $\langle *\rangle$ as its only modal operator (i.e., the fragment of $\mathbf{K}^{*}$ of formulas in which the ordinary 
diamond $\diamond$ does not occur). To overcome the difficulty that $\langle *\rangle$ has direct one-step access to all states in a tree one has to add additional propositional variables to encode the depth of the tree. See the notes for details.

PSPACE upper bound. The $K$-World algorithm for the basic modal system $\mathbf{K}$ (see Figure 7.6) runs in PSPACE. Recall that for any formula $\xi, \xi$ is $\mathbf{K}$ satisfiable iff there exists a set $\Delta \subseteq C l(\xi)$ such that $\xi \in \Delta$ and $K$ - $\operatorname{World}(\Delta, C l(\xi))$ is true. All sets encountered in the execution of $K$-World are subsets of $C l(\xi)$. Each subset of $C l(\xi)$ can be represented in space $O(|\xi|)$, by using pointers to a copy of the formula. Therefore, at each level of the recursion, $O(|\xi|)$ space is used. After $d_{\diamond}(\xi)$ recursive calls $(d \diamond(\xi)$ being the modal depth of $\xi)$, there are no more $\diamond \psi$ formulas in $\Sigma$ and the recursion stops. Thus the recursion depth is bounded by $d_{\diamond}(\xi) \leq|\xi|$, and hence the total amount of space required by the algorithm is $O\left(|\xi|^{2}\right)$. The existential demands in the algorithm (there exists a set $\Delta$ with $\xi \in \Delta$ such that ..., and for all $\diamond \psi \in \Delta$, there exists ...) make the algorithm nondeterministic. But PSPACE $=$ NPSPACE by Savitch's Theorem.

A crucial point in the PSPACE upper-bound argument is that we can represent a complete branch of the tree model for $\xi$ using only polynomial space. Two factors are important here. First, only a polynomial number of formulas is relevant for each world. And second, the depth of the branches is bounded by the modal depth of the input formula. This is caused by the first locality principle: a lack of global expressive power. In the next subsection we show that adding such expressive power to the basic modal language destroys this polynomial-depth property.

\subsubsection{EXPTIME and exponentially deep paths}

Now we shall see that global expressive power in combination with another diamond destroys the polynomially bounded deptLh of the satisfying tree models for the basic modal system. In particular, we shall create a satisfiable formula which, when satisfied, forces a branch in the model containing an exponential number of colors. Thus the PSPACE algorithm sketched in the previous subsection will not work anymore. In fact, the additional expressive power will be enough to show that the satisfiability problem is EXPTIMEhard. We again consider the system $\mathbf{K}^{*}$ of subsection 7.4.2. We want to show that its language is strong enough to force the existence of exponentially deep $R$-paths. A simple way of doing so employs binary counters.

By a binary counter we mean a device that can have a natural number as its value, represented as a binary string of $0 \mathrm{~s}$ and $1 \mathrm{~s}$; it should also be possible to increment this value by one. We use a set $\left\{p_{0}, \ldots, p_{n-1}\right\}$ of propositional variables to implement an $n$-ary binary counter (" $n$-ary" means that the counter is reset to zero after reaching the value $2^{n}-1$ ). We use these variables to encode the $n$ bits of the counter, with $p_{0}$ encoding the least significant and $p_{n-1}$ the most significant bit. The variable $p_{i}$ being true in a given state, 
encodes the fact that the $i$ th bit of the counter is 1 in that state. The key idea of an encoding into the modal language lies in the following characterization of adding 1 to a binary counter. If $a=a_{n-1} \ldots a_{0}$ and $b=b_{n-1} \ldots b_{0}$ are two $n$-bit counters, then $b=a+1\left(\bmod 2^{n}\right)$ precisely when the following holds: either $b_{i}=0$ and $a_{i}=1$ for all $i$ (this is when we start counting at 0 again), or, for some $k \leq n-1$, we have

(1) $a_{k}=0$, and $b_{k}=1$,

(2) $a_{j}=1$ and $b_{j}=0$ for all $j<k$, and

(3) $a_{i}=b_{i}$ for all $i>k$.

In a picture:

$$
\begin{aligned}
& 1011001111 a \\
& 0000000001 \\
& \frac{1011010000 b=a+1 .}{k}
\end{aligned}
$$

We want to write a formula $\gamma(n)$ which forces a counter to take all values from 0 to $2^{n}-1$, in consecutive states, thereby forcing an exponentially deep path. We shall take care that the formula has a length of only $O\left(n^{2}\right)$. The formula $\gamma(n)$ is a conjunction of four formulas. The first conjunct expresses the fact that the counter is initially set to 0 :

$$
\neg p_{0} \wedge \ldots \wedge \neg p_{n-1} .
$$

The other conjuncts of $\gamma(n)$ must hold globally in a model. To achieve that aim, we use the dual $[*]$ of $\langle *\rangle([*] \varphi$ abbreviates $\neg\langle *\rangle \neg \varphi)$. It is clear that the root $s$ of a tree model has $\mathcal{M}, s \Vdash[*] \varphi$ if and only if, for all $t, \mathcal{M}, t \Vdash \varphi$.

The second conjunct expresses that every state has a successor:

$$
[*] \diamond \top \text {. }
$$

The next two conjuncts take care of addition. They express that whenever an $R$-transition is made in the model, the binary counter is increased by one. First, we deal with the simple case of resetting the counter:

$$
[*]\left(\left(p_{0} \wedge \ldots \wedge p_{n-1}\right) \rightarrow \square\left(\neg p_{0} \wedge \ldots \wedge \neg p_{n-1}\right)\right) .
$$

Finally, the last conjunct of $\gamma(n)$ covers the case when we have to "carry one". This conjunct is itself a conjunction, having a conjunct of the following form for every $k$ such that $0 \leq k<n$ :

$$
[*]\left(\left(\neg p_{k} \wedge \bigwedge_{j<k} p_{j}\right) \rightarrow \square\left(p_{k} \wedge \bigwedge_{j<k} \neg p_{j}\right) \wedge \bigwedge_{i>k} \operatorname{store}\left(p_{i}\right)\right),
$$

where $\operatorname{store}\left(p_{i}\right)$ is defined in the previous subsection and the empty conjunction is set to true.

We leave it to the reader to check the correctness of this formula. Note that the sole use of $[*]$ was to make statements in the basic modal language 
true everywhere in the model. This use is crucial, however: Proposition 7.3.10 states that a formula in the basic modal language can only force models with $R$-paths of at most its modal depth. Now the modal depth of $\gamma(n)$ is just two (one for [*], and one for $\diamond$ ), for every $n$, while the minimal $R$-depth of models satisfying $\gamma(n)$ is $2^{n}$.

Complexity bounds. In the previous subsection, we saw that the polynomially bounded depth of models was the key to a PSPACE upper bound. The present result does not yet show that such an upper bound is not possible, but it renders it unlikely (see the notes). And, indeed, the satisfiability problem for $\mathbf{K}^{*}$ is EXPTIME-complete. For the lower bound we refer to the notes. The upper bound follows from the $\mathbf{K}^{*}$ decision algorithm presented in the previous section. Recall that the algorithm tried to construct a set of ready subsets of $C l(\xi)$. We remarked that the construction would terminate after $2^{O(|\xi|)}$ stages. Computing which sets in $S_{i}$ are ready can be done in time polynomial in the size of $S_{i}$, which is at most exponential in $|\xi|$. Thus the whole construction can be carried out in deterministic exponential time.

We note that all results carry over to the modal system $\mathbf{K}+\mathbf{E}$ (the basic modal system expanded with the universal modality E). In particular, the formula $\gamma(n)$ with $\neg\langle *\rangle \neg$ substituted by $\neg \mathrm{E} \neg$ causes an exponentially deep path. This result will be used in the next section.

\subsubsection{NEXPTIME}

We now consider a modal system in which matters get even worse: the system $\mathbf{S 5}^{\mathbf{2}}$. For the definitions of $\mathbf{S 5}^{\mathbf{2}}$ and its square and pseudo-square models, see Section 7.4.1.

This system does not have the tree model property. In addition, the language has global expressive power: for every $\varphi$, if a model $\mathcal{M}$ satisfies $\square_{1} \square_{0} \varphi$, then $\mathcal{M} \Vdash \varphi$. So we expect that the satisfiability problem will have a high complexity. This is indeed the case. This system is strong enough to interpret the system $\mathbf{K}+\mathbf{E}$ of the previous subsection, inheriting its EXPTIME lower bound. But $\mathbf{S 5}^{\mathbf{2}}$ lacks the tree-like models of $\mathbf{K}+\mathbf{E}$ on which the EXPTIME

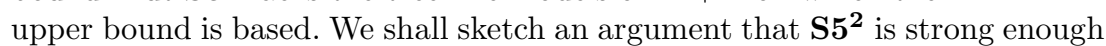
to force exponential grids, which is the key to a NEXPTIME lower-bound result. A matching upper bound follows from earlier results: every satisfiable formula $\xi$ is satisfiable in a pseudo-square of size at most $2^{|\xi|}$, by Proposition 7.4.3. Being a pseudo-square is a first-order property. As we saw in the subsection on NP, testing whether a modal formula is satisfied in a model takes time polynomial in the formula and the size of the model. Thus for the same reasons as why the polysize model property leads to an NP upper bound, we obtain here a NEXPTIME upper bound.

We start with the interpretation of the $\mathbf{K}+\mathbf{E}$ satisfiability problem. For this purpose, we use a translation reminiscent of the two-variable version of the standard translation (read $r$ as $R x y, \diamond_{1}$ as $\exists y$, and $\diamond_{0}$ as $\exists x$, and read 
$w$ as the assertion expressing that $x=y$ ). Let $(\cdot)^{t}$ be a translation function which maps propositional variables to propositional variables, commutes with the booleans, and translates the diamonds as follows:

$$
\begin{aligned}
& (\diamond \varphi)^{t}=\diamond_{1}\left(r \wedge \diamond_{0}\left(w \wedge \varphi^{t}\right)\right) \\
& (\mathrm{E} \varphi)^{t}=\diamond_{1} \diamond_{0}\left(w \wedge \varphi^{t}\right),
\end{aligned}
$$

where $r$ and $w$ are fixed variables not occurring in the input language. Their function becomes clear in the proof of the next proposition.

Proposition 7.5.3. Let $\xi$ be a formula in the basic modal language expanded with the universal modality. Then $\xi$ is $\mathbf{K}+\mathrm{E}$-satisfiable if and only if $w \wedge \xi^{t}$ is $\mathbf{S 5}^{\mathbf{2}}$-satisfiable.

Proof. $(\Rightarrow)$ Let $\mathcal{M}=(W, R, V)$ be a Kripke model and let $\mathcal{M}, s \Vdash \xi$. We define a square $\mathbf{S} 5^{2}$-model $\mathcal{M}^{*}=\left(W \times W, \equiv_{0}, \equiv_{1}, V^{*}\right)$, with $V^{*}$ defined as follows:

$$
\begin{aligned}
& V^{*}(w)=\{(x, x) \mid x \in W\} \\
& V^{*}(r)=R \\
& V^{*}(p)=\{(x, x) \mid x \in V(p)\} .
\end{aligned}
$$

An easy induction shows that $\mathcal{M}^{*},(s, s) \Vdash w \wedge \xi^{t}$.

$(\Leftarrow)$ Let $\mathcal{M}=\left(W, \equiv_{0}, \equiv_{1}, V\right)$ be a square $\mathbf{S} 5^{2}$ model and $\mathcal{M},(s, t) \Vdash w \wedge \xi^{t}$. Define a Kripke model $\mathcal{M}^{\circ}$ whose domain consists of all pairs in $W$ where $w$ holds; of which the valuation $V^{\circ}$ is simply the restriction of $V$ to these $w$-pairs; and in which the accessibility relation $R^{\circ}$ holds between $(x, y)$ and $\left(x^{\prime}, y^{\prime}\right)$ iff $\mathcal{M},\left(x, y^{\prime}\right) \Vdash r$. A simple induction shows that for all $w$-pairs $(x, y)$ and for all formulas $\varphi$, we have that $\mathcal{M},(x, y) \Vdash \varphi^{t} \leftrightarrow \mathcal{M}^{\circ},(x, y) \Vdash \varphi$.

This result immediately shows that the $\mathbf{S 5}^{\mathbf{2}}$ satisfiability problem is EXPTIME-hard. In fact, it is even hard for NEXPTIME. This lower bound can be shown by a reduction to a tiling problem very similar to the one used to show undecidability in Section 7.4. In this case we tile not the grid $\mathbb{N} \times \mathbb{N}$ but the finite grid $2^{n} \times 2^{n}$. It is known that this problem is complete for nondeterministic time exponential in $n$. Here we provide the key idea underlying the reduction, which is that for every $n$, we can define a satisfiable formula $\xi(n)$ with the properties that

- the length of $\xi(n)$ is quadratic in $n$, and

- if $\xi(n)$ is satisfied in an $\mathbf{S 5}^{\mathbf{2}}$ model $\mathcal{M}$, then $\mathcal{M}$ contains as a substructure an isomorphic copy of the structure $\left(2^{n} \times 2^{n}, S_{v}, S_{h}\right)$, where $S_{v}, S_{h}$ are the vertical and horizontal successor functions in the grid $2^{n} \times 2^{n}$.

Once we have expressed this, it is straightforward to find a formula saying that a tiling exists, just as in Section 7.4. Because of space limitations we can give only a very rough sketch. The first conjunct of $\xi(n)$ is the translation of the formula $\gamma(2 n)$ of the previous subsection. We assume that $\gamma(2 n)$ is created from variables $x_{0}, \ldots, x_{n-1}$ and $y_{0}, \ldots, y_{n-1}$. So there are two binary counters, that together specify in binary notation a pair $\langle k, l\rangle$ in the grid $2^{n} \times 2^{n}$. 
Let $\mathcal{M}$ be a Kripke model such that $\mathcal{M}, s \Vdash \gamma(2 n)$. Let $\mathcal{M}^{*}$ be the square $\mathbf{S 5}^{\mathbf{2}}$ model as defined in the proof of Proposition 7.5.3. In $\mathcal{M}^{*}$ we have, besides the tree structure of the Kripke model, also all "grid points": that is, for all worlds $w, w^{\prime} \in W$, the pair $\left(w, w^{\prime}\right)$ exists in the model $\mathcal{M}^{*}$. We can use these pairs to relate the counter information in $w$ and $w^{\prime}$. More concretely, we write formulas ensuring that at $\left(w, w^{\prime}\right)$, a propositional variable $S_{v}$ holds if and only if $w$ encodes the grid pair $\langle k, l\rangle$ and $w^{\prime}$ encodes its vertical successor $\langle k, l+1\rangle$. We again use the characterization of adding one in binary in order to create a formula of the required small size. For the full proof, we refer to the notes.

We have seen in Proposition7.5.3 how the EXPTIME modal logic $\mathbf{K}+\mathbf{E}$ "lives" inside the NEXPTIME logic $\mathbf{S} 5^{2}$. Also, we saw that the "extra" points available in the grid models lead to higher complexity. In the next section, we shall do the same but with more variables. We look at first-order logic and find decidable fragments living inside it. In analogy with the last result, we can say that the key feature of these fragments is that they cannot speak about the "extra grid points".

\subsubsection{Notes}

Most complexity-theoretic classifications of modal satisfiability and validity problems come from the computer science literature. This work can be roughly divided into three groups: temporal logics describing computations, logics for reasoning about knowledge, and description logics. Pointers to this vast literature can be found in the handbook articles by Stirling [56] and Calvanese et alii [13] for temporal logics and description logics, respectively, and in the monograph by Fagin et alii [17] for epistemic logics. Here we provide only the sources for the results in this section.

The NP-completeness of S5 was proved by Ladner [37]. The results on PSPACE come also from [37]: both the upper and the lower bound for the basic modal system $\mathbf{K}$ are established there. Ladner's procedure for $\mathbf{K}$ is like the one given in Figure 7.6, save that he uses "concrete tableaux" (that is, his algorithm specifies how to construct the required atoms) rather than "abstract tableaux" (which factor out the required boolean reasoning). Concrete tableaux were also used by Halpern and Moses [23] to construct PSPACE algorithms for multimodal versions of $\mathbf{K}, \mathbf{S 4}$, and indeed $\mathbf{S 5}$; as these authors show, logics containing two S5 modalities are PSPACE-hard.

The EXPTIME-hardness of $\mathbf{K}^{*}$ and $\mathbf{K}+\mathbf{E}$ is due to Fischer \& Ladner [18] (who work in the richer setting of propositional dynamic logic). An EXPTIME procedure for PDL using an "elimination algorithm" was given by Pratt [50]. We used this idea in the proof of Theorem 7.4.5, and shall do so again in the next section on the guarded fragment. For other applications of the method, see for instance [23], where Halpern \& Moses apply it to a multimodal logic equipped with a common knowledge operator.

The modal system Func+E is an example of a modal system in which exponentially deep paths can be forced but which is still decidable in PSPACE, a 
result due to Sistla \& Clarke [54]. We stress that adding the universal modality causes the complexity to go up from NP. Note that in this system there are no models which can be considered to be binary trees. Indeed, Spaan [55] has provided a sufficient condition for EXPTIME-hardness of the satisfiability problem of modal systems. This criterion requires the existence of models which can be considered as finite binary trees, and an expansion of the basic modal language which is powerful enough to make statements which hold everywhere in such a tree model.

The fact that the square tiling problem with a width given in binary is hard for nondeterministic exponential time was established by Fürer [19]. The lower bound for $\mathbf{S 5}^{\mathbf{2}}$ was established by Marx [40].

\subsection{Modal Logic and First-Order Logic}

The previous sections were centered around the question of what determines the decidability and complexity of the satisfiability problem for various modal systems. We identified the looseness property of modal logics as the main principle guiding their nice computational properties; we also met two locality principles that influence the complexity of a modal system. It now seems natural to try and see how far we can push these ideas concerning looseness and locality to larger fragments of first-order logic than the modal fragment formed by the range of the standard translation map. The aim of this section is to identify a number of decidable fragments of first-order logic; that is, sets of first-order formulas for which it is decidable whether a given formula in the subset is satisfiable in some first-order model or not.

Convention. We work in a relational first-order language with equality. Thus the language contains neither constants nor function symbols. For a sequence of variables $\bar{x}=x_{1}, \ldots, x_{n}$, we shall frequently write $\exists \bar{x} \varphi$, which, as usual, has the same meaning as $\exists x_{1} \cdots \exists x_{n} \varphi$. However, we view $\exists \bar{x}$ not as an abbreviation but as a primitive operator. In particular, this means that the subformulas of $\exists \bar{x} \varphi$ are just $\exists \bar{x} \varphi$ itself, together with the subformulas of $\varphi$. By writing $\varphi(\bar{x})$ we indicate that the free variables of $\varphi$ are among $x_{1}, \ldots, x_{n}$.

\subsubsection{Guarded fragments}

In order to find larger "loose" fragments of first-order logic, we reconsider the game-theoretic characterization of the modal fragment of first-order logic. Recall that bisimulations can be defined using a certain two-pebble EhrenfeuchtFraïssé game in which the universal player's moves are restricted in a certain way. We shall analyze these restrictions and implement them in the standard Ehrenfeucht-Fraïssé games for first-order logic; then we shall be ready to push all modal decidability arguments through for these guarded fragments.

Consider once again the bisimulation game from Section 7.3, and the two crucial properties: 
Locality The game is played by moving a window of fixed size (two, in this case) across the models.

Looseness The window can only be placed on parts of the model in which all different points are related by the accessibility relation.

How do we generalize this to first-order logic? We implement the locality principle by considering fragments of first-order logic using a fixed finite number of variables. The looseness principle can be generalized in (at least) two different ways, leading to different fragments of first-order logic. To state these generalizations, we need two notions, both of which are well known in finite model theory.

Definition 7.6.1. Let $\mathcal{M}=(D, I)$ be a model for some first-order language. A tuple $\left(a_{1}, \ldots, a_{n}\right)$ of objects in $D$ is called live in $\mathcal{M}$ if either $a_{1}=\cdots=a_{n}$ or $\left(a_{1}, \ldots, a_{n}\right) \in I(P)$ for some predicate symbol $P$.

$A$ subset $A$ of $D$ is called guarded if there is some live tuple $\left(a_{1}, \ldots, a_{n}\right)$ such that $A \subseteq\left\{a_{1}, \ldots, a_{n}\right\}$. In particular, singleton sets are always guarded; note also that guarded sets are always finite. A is packed or pairwise guarded if it is finite and each of its two-element subsets is guarded.

These notions can help us to incorporate the looseness principle into Ehrenfeucht-Fraïssé games as follows: player $\forall$ can only move pebbles in such a way that all configurations of pebbles that ever occur on the board are placed on guarded or packed sets.

Definition 7.6.2. Let $\mathcal{M}=(D, I)$ and $\mathcal{M}^{\prime}=\left(D^{\prime}, I^{\prime}\right)$ be two models. A partial isomorphism between $\mathcal{M}$ and $\mathcal{M}^{\prime}$ is a bijection $f: A \rightarrow A^{\prime}$ between some subsets $A$ of $D$ and $A^{\prime}$ of $D^{\prime}$ such that, for all predicate symbols $P$ and all tuples $\bar{a}$ in $A$ (of the appropriate length), we have that $\bar{a} \in I(P)$ if and only if $f(\bar{a}) \in I^{\prime}(P)$.

Now, for a partial isomorphism $f: A_{0} \rightarrow A_{0}^{\prime}$ between $\mathcal{M}^{\prime}$ and $\mathcal{M}^{\prime}$, we define the guarded game $\mathcal{G}_{g}\left(\mathcal{M}, \mathcal{M}^{\prime}, f\right)$ as a variant of the familiar EhrenfeuchtFraïsé game. Here, in each round of the game, $\forall$ selects a structure and a guarded set within that structure; $\exists$ responds with a guarded set in the other structure. A match of the game thus gives rise to two sequences $A=A_{0}, A_{1}, \ldots$ and $A^{\prime}=A_{0}^{\prime}, A_{0}^{\prime}, \ldots$ of subsets of $D$ and $D^{\prime}$, respectively. $\exists$ wins this match if there are local isomorphisms $f_{n}: A_{n} \rightarrow A_{n}^{\prime}(n \in \omega)$ such that $f_{0}=f$ and, for each $n, f_{n}$ and $f_{n+1}$ agree on the intersection $A_{n} \cap A_{n+1}$ while their inverses agree on $A_{n}^{\prime} \cap A_{n+1}^{\prime}$.

Now let $\bar{a}$ in $\mathcal{M}$ and $\bar{a}^{\prime}$ in $\mathcal{M}^{\prime}$ be (possibly empty) sequences of elements such that $f\left(a_{i}\right)=a_{i}^{\prime}$ for all $i$. When $\exists$ has a winning strategy in the guarded game $\mathcal{G}_{g}\left(\mathcal{M}, \mathcal{M}^{\prime}, f\right)$ we say that $\bar{a}$ and $\bar{a}^{\prime}$ are g-bisimilar.

The packed game $\mathcal{G}_{p}\left(\mathcal{M}, \mathcal{M}^{\prime}, f\right)$ and the notion of packed bisimilarity are defined in the same way but using packed sets instead of guarded ones.

These restrictions on the moves of player $\forall$ have direct syntactical counterparts in the form of restrictions on quantification: the idea is that we only allow quantification in the form $\exists \bar{x} \varphi$, where $\varphi$ has to meet certain criteria. 
Definition 7.6.3. We say that a formula $\varphi$ packs a set of variables $\left\{x_{1}, \ldots, x_{k}\right\}$ if $\varphi$ is a conjunction of formulas of the form $x_{i}=x_{j}$ or $R\left(x_{i_{1}}, \ldots, x_{i_{n}}\right)$ or $\exists \bar{y} R\left(x_{i_{1}}, \ldots, x_{i_{n}}\right)$ such that for every $x_{i} \neq x_{j}$, there is a conjunct in $\varphi$ in which $x_{i}$ and $x_{j}$ both occur free.

The packed fragment $P F$ is defined as the smallest set of first-order formulas which contains all atomic formulas and is closed under the boolean connectives and under packed quantification. That is, whenever $\psi$ is a packed formula, $\pi$ packs Free $(\pi)$, and Free $(\psi) \subseteq \operatorname{Free}(\pi)$, then $\exists \bar{x}(\pi \wedge \psi)$ is packed as well; $\pi$ is called the guard of this formula. The guarded fragment GF is the subfragment of PF in which we allow only guarded quantification; that is, packed quantification in which the guard $\pi$ is an atomic formula.

$P F_{n}$ and $G F_{n}$ denote the restrictions to $n$ variables and at most $n$-ary predicate symbols of $P F$ and GF, respectively.

When we want to be specific about the free variables occurring in the formulas, we shall often write $\exists \bar{y}(\pi(\bar{x}, \bar{y}) \wedge \psi(\bar{x}, \bar{y}))$ for the quantified packed formulas, tacitly assuming that $\bar{x}$ and $\bar{y}$ do not share any variables.

Typical examples of guarded (and thus also packed) sentences are $\forall x y(R x y \rightarrow R y x), \exists x y(R x y \wedge R y x \wedge(R x x \vee R y y))$, and the standard translation of a formula in the basic modal language (with $R$ functioning as guard). A typical nonexample is $\forall x y z((R x y \wedge R y z) \rightarrow R x z)$ : it is neither guarded nor packed. For an example of a packed formula which is not guarded, consider $\exists x y z((R x y \wedge R x z \wedge R y z) \wedge \neg C x y z)$.

Note that the notion of packedness only places meaningful restrictions on pairs of distinct variables: since the formula $x=x$ packs the set of variables $\{x\}$, the formula $\exists x(x=x \wedge \psi(x))$, (i.e., with a single quantification over the variable $x)$ is a packed formula, at least, provided that $\psi(x)$ is packed. When $\psi(x)$ is guarded, then $\exists x(x=x \wedge \psi(x))$ is also guarded. Since this formula is equivalent to $\exists x \psi(x)$, this shows that packedness allows a fairly mild form of ordinary quantification, namely over formulas with one free variable. A nice corollary of this is that we may perform the standard translation of the universal modality $\mathrm{E}$ within the two-variable guarded fragment:

$$
S T_{x}(\mathrm{E} \varphi)=S T_{y}(\mathrm{E} \varphi)=\exists x\left(S T_{x}(\varphi)\right) \equiv \exists x\left(x=x \wedge S T_{x}(\varphi)\right) .
$$

A similar translation to first-order logic can be defined for the language with the until modality $U$. Its range is the packed fragment with three variables. The interesting clause here is

$$
S T_{x}(U(\varphi, \psi))=\exists y\left(R x y \wedge S T_{y}(\varphi) \wedge \forall z\left((R x z \wedge R z y) \rightarrow S T_{z}(\psi)\right)\right) .
$$

This formula is not packed itself, because in the subformula $\forall z((R x z \wedge R z y) \rightarrow$ $\left.\left.S T_{z}(\psi)\right)\right)$ the guard $R x z \wedge R z y$ does not pack its own free variables $\{x, y, z\}$. But, of course, the formula is equivalent to

$$
\exists y\left(R x y \wedge S T_{y}(\varphi) \wedge \forall z\left((R x z \wedge R z y \wedge R x y) \rightarrow S T_{z}(\psi)\right)\right)
$$


which is packed. It is not hard to convert this example into a proof showing that every formula in the Until language is equivalent to a packed formula. The (adjusted) translation is another example of a packed sentence that is not guarded.

We have defined first-order fragments by incorporating restrictions on the moves in an Ehrenfeucht-Fraïssé game into the syntax. It is obvious that packed formulas are preserved when player $\exists$ has a winning strategy. But, in fact, the fragments precisely characterize the formulas which are invariant under the corresponding games.

Definition 7.6.4. A first-order formula $\varphi(\bar{x})$ is invariant under guarded (packed) bisimulation if, for all g-bisimilar (p-bisimilar, respectively) tuples $\bar{a}$ in $\mathcal{M}$ and $\bar{a}^{\prime}$ in $\mathcal{M}^{\prime}$ we have that $\mathcal{M} \models \varphi[\bar{a}]$ iff $\mathcal{M}^{\prime} \models \varphi\left[\bar{a}^{\prime}\right]$.

Theorem 7.6.5. Let $\xi$ be a first-order formula. The following are then equivalent:

(i) $\xi$ is equivalent to a formula in the packed (guarded) fragment.

(ii) $\xi$ is invariant under packed (guarded) bisimulations.

This theorem can be relativized in the usual way to $n$-variable fragments and the corresponding $n$-pebble games. This is the first analogue of a modal theorem (the Characterization Theorem 7.3.4). In the section on basic modal logic we saw that this theorem allowed us to prove that every satisfiable formula was satisfiable in a tree. These trees were obtained by unraveling or unwinding the model. Analogous notions of unraveling and tree models can be defined for the guarded and packed fragments as well; here, we confine ourselves to the notion of a loose model.

Definition 7.6.6. Let $\mathcal{M}=(D, I)$ be a first-order structure. We call $\mathcal{M}$ a loose model of degree $k \in \mathbb{N}$ if there is some acyclic connected undirected graph $\mathcal{G}=(G, E)$ and a function $f$ mapping nodes of $\mathcal{G}$ to subsets of $D$ of size not exceeding $k$ such that for every live tuple $\bar{s}$ from $\mathcal{M}$, the set $L(\bar{s})=\{k \in$ $G \mid s_{i} \in f(k)$ for all $\left.s_{i}\right\}$, is a nonempty and connected subset of $\mathcal{G}$.

In words, we call a model $\mathcal{M}=(D, I)$ loose if we can associate a connected graph $\mathcal{G}=(G, E)$ with it in the following way. Each node $t$ of the graph corresponds to a small subset $f(t)$ of the model; a good way of thinking about this is that $t$ "describes" $f(t)$. We then require that the graph "covers" the entire model in the sense that any $a \in D$ belongs to one of these sets (this follows from the fact that for any $a \in D$, the "tuple" $a$ is live). The fact that each set $L(\bar{a})$ is connected whenever $\bar{a}$ is live implies that different nodes of the graph will not give contradictory descriptions of the model. Finally, the looseness of the model stems intuitively from the acyclicity of $\mathcal{G}$ and the connectedness of the sets $L(\bar{a})$, because this ensures that when we walk through the graph we may describe different parts of the model, but we never have to worry about returning to the same part once we have left it. Summarizing, we may see the 
graph as a loose, coherent collection of descriptions of local submodels of the model. The loose models are the ones for which we can find such a graph. Note that the degree of a loose model corresponds directly to the second locality principle that we identified at the end of section 7.3.

Now we can announce our second modally flavored theorem: it establishes the loose model property for the packed fragment.

Theorem 7.6.7. Every satisfiable packed formula $\xi$ can be satisfied on a loose model of degree not exceeding the number of variables occurring in $\xi$.

And, as we shall see later on, this property indeed plays a crucial role in the proof of the following result.

Theorem 7.6.8. It is decidable whether a packed formula is satisfiable. In fact, the satisfiability problems for both the guarded and the packed fragment are complete for 2EXPTIME.

The doubly exponential lower bound may raise doubts concerning the relevance of this result. Fortunately, there are some large and very natural fragments for which better bounds may be obtained, and here the second notion of "locality" comes into play. This is because not only does the concept of looseness generalize to these fragments, but we can also give analogous versions for the notion of locality. Recall that we introduced this concept when we saw that the basic modal language could be translated into the two-variable fragment of first-order logic. This suggests that we might try to improve on Theorem 7.6 .8 by considering finite-variable fragments of $P F$ and $G F$. And, indeed, it turns out that "bringing locality into the language" brings down the complexity by one exponent!

In the case of the guarded fragment, we can formulate this result in a nice way, by imposing conditions on the first-order signature rather than on the number of variables used. Recall that the signature of the modal fragment of first-order logic consists of unary relation symbols and one binary symbol. In general, we call a first-order signature $L n$-bounded if all relation symbols in $L$ have arity at most $n$. It is not very difficult to see that every guarded sentence in an $n$-bounded signature can be rewritten using only $n$ variables. Thus, just as in the basic modal case, the signature determines the number of variables. Note that this property is lost for the full packed fragment, as we can pack arbitrarily large sets with binary relations.

In any case, by implementing both looseness and locality in first-order logic we may obtain the following result.

Theorem 7.6.9. Fix a natural number $n$.

(i) The satisfiability problem for formulas in the packed fragment $P F_{n}$ is decidable in EXPTIME.

(ii) Hence, the satisfiability problem for sentences in the guarded fragment in the $n$-bounded signature is decidable in EXPTIME. 
Note that for $n \geq 2$ the satisfiability problem for the guarded fragment $G F_{n}$ is also EXPTIME-hard. This holds by the interpretation of the modal system $\mathbf{K}+\mathbf{E}$ using the standard translation. However, by also implementing the first locality principle (namely no global expressive power) it is even possible to bring the complexity down to PSPACE, see the notes.

Finally, what about finite models? Several subfragments of the packed fragment, including the guarded fragment, are known to have the finite model property. For the full packed fragment, this was an open problem at the time of writing this chapter, but recently, a positive solution to this problem has been obtained. For reasons of space limitations, we cannot go into detail here — see the notes for references.

\subsubsection{Decidability and complexity}

This subsection provides the proofs of all the results mentioned above. The main idea behind the proofs is given by the mosaic method that we met in the decidability proof for the until system. Roughly speaking, this method is based on the idea of deconstructing models into a (modulo isomorphism) finite collection of finite submodels and, conversely, of building up new, "nice", models from such parts.

This subsection is structured as follows. We start with a formal definition of the notion of mosaics and some related concepts. We then state the main result concerning the mosaic method, namely the Mosaic Theorem, stating that a packed formula has a model if and only if there is a bounded set of boundedsize mosaics for it. This enables us to define our decision algorithms and establish their complexity. We then continue by proving the Mosaic Theorem. In doing so, we obtain as a by-product the loose model property for the packed fragment.

\section{Linked sets of mosaics}

Mosaics form the key tools in our proof; for a formal definition we need some syntactic preliminaries. Given a first-order formula $\xi$, we let $\operatorname{Var}(\xi)$ and Free $(\xi)$ denote the sets of variables and free variables, respectively, occurring in $\xi$. Let $V$ be a set of variables. A $V$-substitution is any partial map $\sigma: V \rightarrow V$. The result of performing a substitution $\sigma$ on the formula $\psi$ is denoted by $\psi^{\sigma}$. (We can and may assume that if $\operatorname{Var}(\psi) \subseteq V$, then $\operatorname{Var}\left(\psi^{\sigma}\right) \subseteq V$. For instance, when substituting $y$ for $x$ in $R x z \wedge \forall y(R z y \rightarrow Q x y)$, we have to rename the bound variable $y$, as in $R y z \wedge \forall u(R z u \rightarrow Q y u)$. The point is that we do not need to use a fresh variable $u$ for this: instead, we may reuse $x$, giving $R y z \wedge \forall x(R z x \rightarrow Q y x)$.)

As before, we shall employ a notion of closure to delineate a finite set of relevant formulas, i.e., formulas that for some reason critically influence the truth of a given formula $\xi$. Also, recall that the single negation $\sim \varphi$ of a formula 
$\varphi$ denotes the formula $\psi$ if $\varphi$ is of the form $\neg \psi$; otherwise, $\sim \varphi$ is the formula $\neg \varphi$.

Definition 7.6.10. Let $\Sigma$ be a set of packed formulas in the set $V$ of variables. We call $\Sigma V$-closed if $\Sigma$ is closed under subformulas, single negations, and $V$-substitutions (that is, if $\psi$ belongs to $\Sigma$, then so does $\psi^{\sigma}$ for every $V$-substitution $\sigma)$. By $C l_{g}(\xi)$, we denote the smallest $\operatorname{Var}(\xi)$-closed set of formulas containing $\xi$.

For the remainder of this section, we fix a packed formula $\xi$ - all definitions to come should be understood as being relativized to $\xi$. The number of variables occurring in $\xi$ (free or bound) is denoted by $k$; that is, $k$ is the size of $\operatorname{Var}(\xi)$. It can easily be verified that the sets of guarded and packed formulas are both closed under taking subformulas; hence, the set $\mathrm{Cl}_{g}(\xi)$ consists of guarded (packed, respectively) formulas. An easy calculation shows that the cardinality of $C l_{g}(\xi)$ is bounded by $k^{k} \cdot(2|\xi|)$ ( $k^{k}$ is the number of $\operatorname{Var}(\xi)$-substitutions).

The following notion is the counterpart of the maximally propositionally consistent sets that we have met in earlier decidability proofs. The defining conditions again derive from a desire to prove a truth lemma.

Definition 7.6.11. Let $X \subseteq \operatorname{Var}(\xi)$ be a set of variables. An $X$-type is a set $\Gamma \subseteq C l_{g}(\xi)$ with free variables in $X$ satisfying, for all formulas $\varphi \wedge \psi, \sim \varphi$, and $\varphi$ in $C l_{g}(\xi)$ with free variables in $X$, the following conditions:

(T1) $\varphi \wedge \psi \in \Gamma$ iff $\varphi \in \Gamma$ and $\psi \in \Gamma$;

(T2) $\varphi \notin \Gamma$ iff $\sim \varphi \in \Gamma$;

(T3) $\varphi, x_{i}=x_{j} \in \Gamma$ only if $\varphi^{\sigma} \in \Gamma$ (for any substitution $\sigma$ mapping $x_{i}$ to $x_{j}$ and/or $x_{j}$ to $x_{i}$, while leaving all other variables fixed); and

(T4) if $\psi(\bar{x}, \bar{z})$ and $\pi(\bar{x}, \bar{z})$ are in $\Gamma$, then so is $\exists \bar{y}(\pi(\bar{x}, \bar{y}) \wedge \psi(\bar{x}, \bar{y}))$ (provided that the latter formula belongs to $\left.\mathrm{Cl}_{g}(\xi)\right)$.

The next definition introduces our key tool for proving the decidability of the packed fragment: mosaics and linked sets of mosaics. Basically, a mosaic consists of a set $X$ of variables in $\operatorname{Var}(\xi)$ and a set $\Gamma$ encoding the relevant information about some small part of a model. Here "small" means that its size is bounded by the number of objects that can be named using variables in $X$, and "relevant" refers to all formulas in $C l_{g}(\xi)$ whose free variables are in $X$. It turns out that a finite set of such mosaics contains sufficient information to construct a model for $\xi$, provided that the set links the mosaics together in a nice way. Here is a more formal definition.

Definition 7.6.12. A mosaic is a pair $(X, \Gamma)$ such that $X \subseteq \operatorname{Var}(\xi)$ and $\Gamma \subseteq C l_{g}(\xi)$. A mosaic $(X, \Gamma)$ is coherent if $\Gamma$ is an $X$-type.

$A$ link between two mosaics $(X, \Gamma)$ and $\left(X^{\prime}, \Gamma^{\prime}\right)$ is a renaming (that is, an injective substitution) $\sigma$ with $\operatorname{dom}(\sigma) \subseteq X$ and $\operatorname{ran}(\sigma) \subseteq X^{\prime}$ which satisfies, for all formulas $\varphi \in C l_{g}(\xi), \varphi \in \Gamma$ iff $\varphi^{\sigma} \in \Gamma^{\prime}$. 
$A$ requirement of a mosaic is a formula of the form $\varphi(\bar{x})=\exists \bar{y}(\pi(\bar{x}, \bar{y}) \wedge$ $\psi(\bar{x}, \bar{y}))$ belonging to $\Gamma$. A mosaic $\left(X^{\prime}, \Gamma^{\prime}\right)$ fulfills the requirement $\exists \bar{y}(\pi(\bar{x}, \bar{y}) \wedge$ $\psi(\bar{x}, \bar{y}))$ of a mosaic $(X, \Gamma)$ via the link $\sigma$, if, for some variables $\bar{u}, \bar{v}$ in $X^{\prime}$, we have that $\sigma(\bar{x})=\bar{u}$ and that $\pi(\bar{u}, \bar{v})$ and $\psi(\bar{u}, \bar{v})$ belong to $\Gamma^{\prime}$. A set $S$ of mosaics is linked if every requirement of every mosaic in $S$ is fulfilled via some link to some mosaic in $S . S$ is a linked set of mosaics for $\xi$ if it is linked and $\xi \in \Gamma$ for some $(X, \Gamma)$ in $S$.

Note that a mosaic $(X, \Gamma)$ may fulfill its own requirements, either via the identity map or via some other map from $X$ to $X$.

The key result concerning mosaics is the following Mosaic Theorem.

Theorem 7.6.13 (Mosaic Theorem). Let $\xi$ be a packed formula. Then $\xi$ is satisfiable if and only if there is a linked set of mosaics for $\xi$.

Proof. The hard, right-to-left, direction of the theorem is proved in Lemma 7.6.14 below; here we prove only the other direction.

Suppose that $\xi$ is satisfied in the model $\mathcal{M}=(D, I)$. In a straightforward way we can "cut out" from $\mathcal{M}$ a linked set of mosaics for $\xi$. Consider the set of partial assignments of elements in $D$ to variables in $\operatorname{Var}(\xi)$. For each such $\alpha$, let $\left(X_{\alpha}, \Gamma_{\alpha}\right)$ be the mosaic given by $X_{\alpha}=\operatorname{dom}(\alpha)$ and

$$
\Gamma_{\alpha}=\left\{\varphi \in C l_{g}(\xi) \mid \mathcal{M} \models \varphi[\alpha]\right\} .
$$

We leave it to the reader to verify that this collection forms a linked set of mosaics for $\xi$.

In establishing the hard direction of this proposition, we shall in fact prove something stronger: starting from a linked set of mosaics for a formula $\xi$ we shall show that there is a loose or tree-like model for $\xi$.

First, however, we want to show that the Mosaic Theorem is the key for proving the decidability of the packed fragment, and also for finding an upper bound for its complexity.

\section{The decision algorithm and its complexity}

The Mosaic Theorem tells us that any packed formula $\xi$ is satisfiable if and only if there is a linked set of mosaics for $\xi$. Thus in order to decide whether $\xi$ is satisfiable, it suffices to give an algorithm which decides the existence of a linked set of mosaics for $\xi$. We shall establish the upper complexity bound for the satisfiability problem of packed formulas by implementing such an algorithm. The following observations are easy consequences of our definitions; recall that $k$ denotes the number of variables occurring in $\xi$.

- We have already observed that the cardinality of $C l_{g}(\xi)$ is bounded by $k^{k} \cdot 2|\xi|$. 
- The number of mosaics does not exceed $2^{k} \cdot 2^{2|\xi| \cdot k^{k}}$; using the big $O$ notation, this gives at most $2^{O(|\xi|) \cdot k^{k}}$ mosaics.

- given sets $X, \Gamma$ with $X \subseteq \operatorname{Var}(\xi)$ and $\Gamma \subseteq C l_{g}(\xi)$, it is decidable in time $2^{O(|\xi|) \cdot k^{k}}$ whether $(X, \Gamma)$ is a coherent mosaic.

Our algorithm is very similar to the one we used for the until system in subsection 7.4.3. Let $S_{0}$ be the set of all coherent mosaics. By the observations above, $S_{0}$ contains fewer than $2^{O(|\xi|) \cdot k^{k}}$ elements and can be constructed in time $2^{O(|\xi|) \cdot k^{k}}$. We now inductively construct a sequence of sets of mosaics $S_{0} \supsetneq S_{1} \supsetneq S_{2} \supsetneq S_{3} \cdots$, as follows. We call a mosaic $\mu$ in a set $S_{i} S_{i}$-ready if each of its requirements is fulfilled in (some mosaic of) $S_{i}$. Note that one can determine the $S_{i}$-readiness of a mosaic $(X, \Gamma)$ by checking, for each requirement $\varphi(\bar{x}) \in \Gamma$, whether there is a link $\sigma$ to some mosaic $\left(X^{\prime}, \Gamma^{\prime}\right) \in S_{i}$ which fulfills the requirement. If every mosaic $\mu$ in $S_{i}$ is $S_{i}$-ready, then return "YES" if $S_{i}$ contains a mosaic $(X, \Gamma)$ with $\xi \in \Gamma$, and "NO" if $S_{i}$ contains no such mosaic. If, on the other hand, there are mosaics in $S_{i}$ that are not $S_{i}$-ready, then we let $S_{i+1}$ consist of the $S_{i}$-ready mosaics and continue the algorithm.

Clearly the algorithm is correct; and since $S_{i} \supsetneq S_{i+1}$, the construction must halt after at most $\left|S_{0}\right|$ many stages. So let us now see about the complexity. At each stage $i$, the algorithm determines the $S_{i}$-ready mosaics; we claim that this can be done in time exponential in $k^{k} \cdot O(|\xi|)$.

To check whether a given link between two given mosaics fulfills some given requirement is a task that takes time linear in the size of each mosaic, and so time quadratic in $k^{k} \cdot 2|\xi|$. In order to find out whether a given mosaic $(X, \Gamma)$ in a set $S_{i}$ is $S_{i}$-ready, the algorithm has to check, for every requirement $\varphi(\bar{x})$ of the mosaic, for every link $\sigma$, and for every mosaic $\left(X^{\prime}, \Gamma^{\prime}\right)$ in $S_{i}$, whether $\sigma$ is a link between the mosaics fulfilling the requirement. Clearly, then, for a given mosaic, this takes time at most $k^{k} \cdot 2|\xi|$ (for the number of requirements) times $k^{k}$ (for the number of links) times $\left|S_{i}\right|$ (for the number of mosaics) times $\left(k^{k} \cdot 2|\xi|\right)^{2}$ (for the checking time). Note that $S_{i}$ is the only number in this product that is exponential in $k^{k} \cdot O(|\xi|)$. Hence, in order to compute all the $S_{i}$-ready mosaics, the algorithm needs time exponential in $O(|\xi|) \cdot k^{k}$.

As the size of $S_{0}$ is bounded by $2^{O(|\xi|) \cdot k^{k}}$, the whole computation can be performed in time exponential in $O(|\xi|) \cdot k^{k}$. Hence, if we consider a formula $\xi$ in a packed fragment with a fixed number of variables, $\left|S_{0}\right|$ is singly exponential in $|\xi|$. In general, however, the number of variables $k$ occurring in a formula depends on the formula's length and hence, in general, $\left|S_{0}\right|$ is doubly exponential in $|\xi|$. Thus, pending the proof of the next lemma, this shows the upper complexity bounds given in Theorems 7.6.8 and 7.6.9.

\section{Step-by-step construction and loose models}

We now show the hard direction of the Mosaic Theorem and establish, as a by-product, the "loose model property" of Theorem 7.6.7. 
Lemma 7.6.14. Let $\xi$ be a packed formula. If there is a linked set of mosaics for $\xi$, then $\xi$ is satisfiable in a loose model of degree $|\operatorname{Var}(\xi)|$.

Proof. Assume that $S$ is a linked set of mosaics for $\xi$. Using a step-by-step construction, we shall build a model for $\xi$, together with a graph $\mathcal{G}$ and a function $f$ mapping nodes of $\mathcal{G}$ to subsets of the domain of the model. At each stage of the construction, we shall be dealing with some kind of approximation of the final model and graph; these approximations will be called networks and are fairly complex structures.

A network is a quintuple $(\mathcal{M}, \mathcal{G}, \mu, \alpha, \sigma)$ such that $\mathcal{M}=(D, I)$ is a model for the first-order language; $\mathcal{G}=(G, E)$ is a connected, adirected, and acyclic graph; $\mu: G \rightarrow S$ is a map associating a mosaic $\mu_{t}=\left(X_{t}, \Gamma_{t}\right)$ in $S$ with each node $t$ of the graph; and $\alpha$ is a map associating a map $\alpha_{t}: X_{t} \rightarrow D$ with each node $t$ of the graph. (This map is thus a partial assignment of the variables occurring in $\xi$.) And, finally, $\sigma$ is a map associating with each edge $\left(t, t^{\prime}\right)$ of the graph a link $\sigma_{t t^{\prime}}$ from $\mu_{t}$ to $\mu_{t^{\prime}}$ (we shall usually simplify our notation by writing $\sigma$ instead of $\sigma_{t t^{\prime}}$ ).

The idea is that each mosaic $\mu_{t}$ is supposed to give a complete description of the relevant requirements that we impose on a small part of the model-tobe. Which part? This is given by the assignment $\alpha_{t}$. And the word "relevant" refers to the fact that we are interested only in the formulas influencing the truth of $\xi$; that is, the formulas in $C l_{g}(\xi)$. The links between neighboring mosaics are there to ensure that distinct mosaics agree on the part of the model that they both have access to.

Now, obviously, if we want all of this to work properly we have to impose some conditions on the networks. In order to formulate these, we need some auxiliary notation. For a subset $A \subseteq D$, let $L(A)$ denote the set of nodes in $\mathcal{G}$ that have "access" to $A$; formally, we define $L(A)=\left\{t \in G \mid A \subseteq \operatorname{ran}\left(\alpha_{t}\right)\right\}$. For a tuple $\bar{a}=\left(a_{1}, \ldots, a_{n}\right)$ of elements in $D$ we set $L(\bar{a})=L\left(\left\{a_{1}, \ldots, a_{n}\right\}\right)$. Now a network is called coherent if it satisfies the following conditions (all to be read as universally quantified):

(C1) $P \bar{x} \in \Gamma_{t}$ iff $\mathcal{M}=P \bar{x}\left[\alpha_{t}\right]$;

(C2) $x_{i}=x_{j} \in \Gamma_{t}$ iff $\alpha_{t}\left(x_{i}\right)=\alpha_{t}\left(x_{j}\right)$;

(C3) $L(A)$ is nonempty for every guarded set $A \subseteq D$;

(C4) $L(A)$ is connected for every guarded set $A \subseteq D$;

(C5) if $E t t^{\prime}$, then $\sigma_{t t^{\prime}}(x)=x^{\prime}$ iff $\alpha_{t}(x)=\alpha_{t^{\prime}}\left(x^{\prime}\right)$.

A few words of explanation about these conditions: (C1) and (C2) ensure that every mosaic is a complete description of the atomic formulas that hold in the part of the model it refers to. Condition (C3) states that no guarded set in the model remains unseen from the graph, and the conditions (C4) and (C5) are the crucial ones that ensure that remote parts of the graph cannot contain contradictory information about the model - how this works precisely will become clear later on. Note that condition (C5) has two directions: the left-toright direction states that neighboring mosaics have common access to part of 
the model, while the other direction makes them agree on their requirements concerning this common part.

The motivation for using these networks is that in the end we want any formula $\varphi(\bar{x}) \in C l_{g}(\xi)$ to hold in $\mathcal{M}$ under the assignment $\alpha_{t}$ if and only if $\varphi(\bar{x})$ belongs to $\Gamma_{t}$. Coherence on its own is not sufficient to make this happen. A defect of a network consists of a formula $\exists \bar{y}(\pi(\bar{x}, \bar{y}) \wedge \psi(\bar{x}, \bar{y}))$ which is a requirement of the mosaic $\mu_{t}$ for some node $t$ while there is no neighboring node $t^{\prime}$ such that $\mu_{t^{\prime}}$ fulfills $\exists \bar{y}(\pi(\bar{x}, \bar{y}) \wedge \psi(\bar{x}, \bar{y}))$ via the link $\sigma_{t t^{\prime}}$. A coherent network $\mathcal{N}$ is perfect if it has no defects. We say that $\mathcal{N}$ is a network for $\xi$ if for some $t \in G, \mu_{t}=\left(X_{t}, \Gamma_{t}\right)$ is such that $\xi \in \Gamma_{t}$.

Claim 1. If $\mathcal{N}=(\mathcal{M}, \mathcal{G}, \mu, \alpha, \sigma)$ is a perfect network, then

(i) $\mathcal{M}$ is a loose model of degree $|\operatorname{Var}(\xi)|$, and

(ii) for all formulas $\varphi(\bar{x}) \in C l_{g}(\xi)$ and all nodes $t$ of $\mathcal{G}$,

$$
\varphi \in \Gamma_{t} \text { iff } \mathcal{M} \models \varphi\left[\alpha_{t}\right]
$$

Proof of Claim For part (i) of the claim, let $\mathcal{N}=(\mathcal{M}, \mathcal{G}, \mu, \alpha, \sigma)$ be the perfect network for $\xi$. Let $\mathcal{M}=(D, I)$. As the function $f$ mapping nodes of $\mathcal{G}$ to subsets of $D$, we simply take the map that assigns the range of $\alpha_{t}$ to the node $t$. Since the domain of each map $\alpha_{t}$ is always a subset of $\operatorname{Var}(\xi)$, it follows immediately that $f(t)$ will always be a set of size at most $|\operatorname{Var}(\xi)|$. Now take an arbitrary live tuple $\bar{s}$ in $\mathcal{M}$; it follows from (C3) and (C4) that $L(\bar{s})$ is a nonempty and connected part of the graph $\mathcal{G}$. Thus $\mathcal{M}$ is a loose model of degree $|\operatorname{Var}(\xi)|$.

We prove part (ii) of the claim by induction on the complexity of $\varphi$. For atomic formulas, the claim follows by conditions (C1) and (C2), and the boolean case of the induction step is straightforward (since $\Gamma_{t}$ is an $X$-type) and is left to the reader. We concentrate on the case where $\varphi(\bar{x})$ is of the form $\exists \bar{y}(\pi(\bar{x}, \bar{y}) \wedge \psi(\bar{x}, \bar{y}))$.

First, assume that $\varphi(\bar{x}) \in \Gamma_{t}$. Since $\mathcal{N}$ is perfect there is a node $t^{\prime}$ in $G$ and variables $\bar{u}, \bar{v}$ in $X_{t^{\prime}}$ such that $E t t^{\prime}, \pi(\bar{u}, \bar{v})$, and $\psi(\bar{u}, \bar{v})$ belong to $\Gamma_{t^{\prime}}$, while the link $\sigma$ from $\mu_{t}$ to $\mu_{t^{\prime}}$ maps $\bar{x}$ to $\bar{u}$. By the induction hypothesis, we find that

$$
\mathcal{M} \models \pi(\bar{u}, \bar{v}) \wedge \psi(\bar{u}, \bar{v})\left[\alpha_{t^{\prime}}\right] .
$$

But, from condition (C5), it follows that $\alpha_{t^{\prime}}(\bar{x})=\alpha_{t}(\bar{u})$; hence (7.13) implies that

$$
\mathcal{M} \models \exists \bar{y}(\pi(\bar{x}, \bar{y}) \wedge \psi(\bar{x}, \bar{y}))\left[\alpha_{t}\right],
$$

which is what we were after.

Now suppose, in order to prove the converse direction, that $\mathcal{M}=\varphi(\bar{x})\left[\alpha_{t}\right]$. Let $\bar{a}$ denote $\alpha_{t}(\bar{x})$; there are then $\bar{b}$ in $D$ such that $\mathcal{M} \models \pi(\bar{x}, \bar{y})[\bar{a} \bar{b}]$ and $\mathcal{M} \models \psi(\bar{x}, \bar{y})[\bar{a} \bar{b}]$. Our first aims are to prove that

$$
L(\bar{a} \bar{b}) \neq \varnothing
$$

and 


$$
L(A) \text { is connected for every } A \subseteq\{\bar{a}, \bar{b}\} .
$$

Note that if we are working in the guarded fragment, then $\pi(\bar{x}, \bar{y})$ is an atomic formula, and hence it follows from $\mathcal{M}=\pi(\bar{x}, \bar{y})[\bar{a} \bar{b}]$ that $\bar{a} \bar{b}$ is live. Thus $\{\bar{a}, \bar{b}\}$ is guarded, and hence (7.14) follows directly by condition (C3). In fact, every $A \subseteq\{\bar{a}, \bar{b}\}$ is guarded in this case, and so (7.15) follows immediately by condition ( $\mathrm{C} 4)$.

In the more general case of the packed fragment we have to work a little harder. First, observe that it does follow from $\mathcal{M} \models \pi(\bar{x}, \bar{y})[\bar{a} \bar{b}]$ and the conditions on $\pi(\bar{x}, \bar{y})$ in the definition of packed quantification that $\{c, d\}$ is guarded, and thus $L(c, d) \neq \varnothing$, for every pair $(c, d)$ of points taken from $\bar{a} \bar{b}$. It follows from (C4) that $\{L(c, d) \mid c, d$ taken from $\bar{a} \bar{b}\}$ is a collection of nonempty, connected, pairwise overlapping subgraphs of the acyclic graph $\mathcal{G}$. It is fairly straightforward to prove, for instance by induction on the size of the graph $\mathcal{G}$, that any such collection must have a nonempty intersection. From this, (7.14) and (7.15) follow almost immediately.

We thus may assume the existence of a node $t^{\prime}$ in $\mathcal{G}$ such that $\{\bar{a}, \bar{b}\} \subseteq$ $\operatorname{ran} \alpha_{t^{\prime}}$. Let $\bar{u}$ and $\bar{v}$ in $X_{t^{\prime}}$ be the variables such that $\alpha_{t^{\prime}}(\bar{u})=\bar{a}$ and $\alpha_{t^{\prime}}(\bar{v})=\bar{b}$. The induction hypothesis implies that $\pi(\bar{u}, \bar{v})$ and $\psi(\bar{u}, \bar{v})$ belong to $\Gamma_{t^{\prime}}$, and hence $\varphi(\bar{u}) \in \Gamma_{t^{\prime}}$ by the coherence of $\mu_{t^{\prime}}$. Since both $t$ and $t^{\prime}$ belong to $L(\bar{a})$, it follows from (7.15) that there is a path from $t$ to $t^{\prime}$ within $L(\bar{a})$, say $t^{\prime}=s_{0} E s_{1} E \ldots E s_{n}=t$. Let $\sigma_{i}$ be the link between the mosaics of $s_{i}$ and $s_{i+1}$, and define $\rho$ to be the composition of these maps. It follows by an easy inductive argument on the length of the path that $\rho$ is a link between $\mu_{t^{\prime}}$ and $\mu_{t}$ such that $\rho(\bar{u})=\bar{x}$. Hence, by the definition of a link, we have that $\varphi(\bar{x}) \in \Gamma_{t^{\prime}}$. This finishes the proof of the claim.

By Claim 1, in order to prove the lemma it suffices to construct a perfect network for $\xi$. This construction uses a step-by-step argument; to start the construction, we need some coherent network for $\xi$.

Claim 2. There is a coherent network for $\xi$.

Proof of Claim By our assumption about $\xi$, there is a coherent mosaic $\mu=(X, \Gamma)$ such that $\xi \in \Gamma$. Without loss of generality we may assume that $X$ is the set $\left\{x_{1}, \ldots, x_{n}\right\}$ (otherwise, we can take an isomorphic copy of $\mu$ in which $X$ does have this form). Let $a_{1}, \ldots, a_{n}$ be a list of objects such that, for all $i$ and $j$, we have that $a_{i}=a_{j}$ if and only if the formula $x_{i}=x_{j}$ belongs to $\Gamma$. Define $D=\left\{a_{1}, \ldots, a_{n}\right\}$, and put the tuple $\left(a_{i_{1}}, \ldots, a_{i_{k}}\right)$ in the interpretation $I(P)$ of the $k$-ary predicate symbol $P$ precisely if $P x_{i_{1}} \ldots x_{i_{n}} \in \Gamma$. Let $\mathcal{M}$ be the resulting model $(D, I)$, and define $\mathcal{G}$ as the trivial graph with one node 0 and no edges. Let $\mu(0)$ be the mosaic $\mu$; let $\alpha_{0}: X \rightarrow D$ be given by $\alpha\left(x_{i}\right)=a_{i}$; and, finally, let $\sigma_{00}$ be the identity map from $X$ to $X$.

We leave it for the reader to verify that the quintuple $(\mathcal{M}, \mathcal{G}, \mu, \alpha, \sigma)$ is a coherent network for $\xi$. This finishes the proof of the claim. 
The crucial step of this construction is to show that any defect of a coherent network can be repaired.

Claim 3. For any coherent network $\mathcal{N}=(\mathcal{M}, \mathcal{G}, \mu, \alpha, \sigma)$ and any defect of $\mathcal{N}$ there is a coherent network $\mathcal{N}^{+}$that extends $\mathcal{N}$ and lacks this defect.

Proof of Claim Suppose that $\varphi(\bar{x})$ is a defect of $\mathcal{N}$ because it is a requirement of the mosaic $\mu_{t}$ and not fulfilled by any neighboring mosaic $\mu_{t^{\prime}}$. We shall define an extension $\mathcal{N}^{+}$of $\mathcal{N}$ in which this defect is repaired.

Since $S$ is a linked set of mosaics and $\mu_{t}$ belongs to $S, \mu_{t}$ is linked to a mosaic $\left(X^{\prime}, \Gamma^{\prime}\right) \in S$ in which the requirement is fulfilled via some link $\rho$. Let $Y$ be the set of variables in $X^{\prime}$ that do not belong to the range of $\rho$; suppose that $Y=\left\{y_{1}, \ldots, y_{k}\right\}$ (with all $y_{i}$ being distinct). For the sake of a smooth presentation, assume that $\Gamma^{\prime}$ contains the formulas $\neg x^{\prime}=y$ for all variables $x^{\prime} \in X^{\prime}$ and $y \in Y$ (this is not without loss of generality - we leave the general case as an exercise for the reader). Take a set $\left\{c_{1}, \ldots, c_{k}\right\}$ of fresh objects (that is, no $c_{i}$ is an element of the domain $D$ of $\mathcal{M}$ ), and let $\gamma$ be the assignment with domain $X^{\prime}$ defined as follows:

$$
\gamma\left(x^{\prime}\right)= \begin{cases}\alpha_{t}(x) & \text { if } x^{\prime}=\rho(x), \\ c_{i} & \text { if } x^{\prime}=y_{i} .\end{cases}
$$

Let $t^{\prime}$ be an object not belonging to $G$. Now define the network $\mathcal{N}^{+}=$ $\left(\mathcal{M}^{+}, \mathcal{G}^{+}, \mu^{+}, \alpha^{+}, \sigma^{+}\right)$as follows:

$$
\begin{aligned}
D^{+} & =D \cup\left\{c_{1}, \ldots, c_{k}\right\}, \\
I^{+}(P) & =I(P) \cup\left\{\bar{d} \mid \text { for some } \bar{x}, \bar{d}=\gamma(\bar{x}) \text { and } P \bar{x} \in \Gamma^{\prime}\right\}, \\
G^{+} & =G \cup\left\{t^{\prime}\right\}, \\
E^{+} & =E \cup\left\{\left(t, t^{\prime}\right)\right\},
\end{aligned}
$$

and $\mu^{+}, \alpha^{+}$and $\sigma^{+}$are given by the obvious extensions of $\mu, \alpha$, and $\sigma$, namely by putting $\mu_{t^{\prime}}^{+}=\left(X^{\prime}, \Gamma^{\prime}\right), \alpha_{t^{\prime}}^{+}=\gamma$, and $\sigma_{t t^{\prime}}=\rho$.

Since the interpretation $I^{+}$agrees with $I$ on "old" tuples, it is a straightforward exercise to verify that the new network $\mathcal{N}^{+}$satisfies the conditions (C1 - C3) and (C5).

In order to check that condition (C4) holds, take some guarded subset $A$ from $D^{+}$; we shall show that $L^{+}(A)$ is a connected subgraph of $\mathcal{G}^{+}$. It is rather easy to see that $L^{+}(A)$ is identical to either $L(A)$ or $L(A) \cup\left\{t^{\prime}\right\}$; hence by the connectedness of $L(A)$, it suffices to prove, on the assumption that $t^{\prime} \in L^{+}(A)$ and $L(A) \neq \varnothing$, that $t \in L(A)$. Hence, suppose that $t^{\prime} \in L^{+}(A)$; that is, each $a \in A$ is in the range of $\gamma$. But if $L(A) \neq \varnothing$, each such point $a$ must be old; hence, by the definition of $\gamma$, each $a \in A$ must belong to $\operatorname{ran}\left(\alpha_{t}\right)$. This gives the result that $t \in L(A)$, as required. This finishes the proof of the claim.

As in the proof for the until system, the previous two claims show that by using some standard combinatorics we can construct a chain of networks such that their limit is a perfect network. This finishes the proof of the lemma. 


\subsubsection{Notes}

The roots of the decidability proof in this section date back to 1986, when Németi [45] showed that the equational theory of the class Crs of relativized cylindric set algebras is decidable. The first-order counterpart of this result is that a certain subfragment of the guarded fragment is decidable.

The importance of this result for first-order logic was realized in 1994 when Andréka, van Benthem \& Németi introduced the guarded fragment and showed that many nice properties of the basic modal system $\mathbf{K}$ generalize to it. In particular, these authors established a characterization in terms of guarded bisimulations, decidability, and a kind of tree model property. The journal version of their paper is [2]. Some time later, van Benthem [7] generalized some of the results, introducing the loosely guarded fragment. The slightly more general packed fragment was introduced by Marx [41] in order to give a semantic characterization in terms of packed bisimulations (Theorem 7.6.5). (An example of a packed sentence which is not equivalent to a loosely guarded sentence in the same signature is $\exists x y z(\exists w C x y w \wedge \exists w C x z w \wedge \exists w C z y w \wedge$ $\neg C x y z)$.)

The mosaic-based decision algorithms used by Andréka, van Benthem \& Németi were essentially optimal, a result established by Grädel [21]. In that paper, Grädel also defind and established the loose model property for the loosely guarded fragment. Our definition of a loose model is based on the definition of a tree model given there. Grädel \& Walukiewicz [22] showed that the same bounds obtain when the guarded fragment is expanded with least and greatest fixed-point operators. Marx, Schlobach \& Mikulas [42] defined a PSPACE complete guarded fragment with the finite tree model property. This fragment satisfies both locality principles.

The finite model property for the guarded fragment and several subfragments of the packed fragment was established in an algebraic setting by Andréka, Hodkinson \& Németi [1]. Grädel [21] provided a direct proof for the guarded fragment. After we finished the writing of this chapter, Hodkinson [32] proved the finite model property of the full packed fragment. All these results are based on variants of a result due to Herwig [29]. The use of Herwig's Theorem to establish the finite model property and to eliminate the need of step-by-step constructions originates with Hirsch et alii [30].

\section{Acknowledgments}

We are very grateful to Carlos Areces, Edith Hemaspaandra, and Carla Piazza for scrutinizing earlier versions of this manuscript and for making many suggestions for improvement. We would also like to thank Moshe Vardi and Scott Weinstein for inviting us to participate in this project. 


\section{References}

1. H. Andréka, I. Hodkinson, and I. Németi. Finite algebras of relations are representable on finite sets. Journal of Symbolic Logic, 64(1):243-267, 1999.

2. H. Andréka, J. van Benthem, and I. Németi. Modal languages and bounded fragments of predicate logic. Journal of Philosophical Logic, 27(3):217-274, 1998.

3. J. Balcázar, J. Díaz, and J. Gabarró. Structural Complexity I. EATCS Monographs on Theoretical Computer Science, No. 11. Springer, 1988.

4. J. van Benthem. Modal Correspondence Theory. PhD thesis, Mathematisch Instituut \& Instituut voor Grondslagenonderzoek, University of Amsterdam, 1976.

5. J. van Benthem. Modal Logic and Classical Logic. Bibliopolis, Naples, 1983.

6. J. van Benthem. Exploring Logical Dynamics. Studies in Logic, Language and Information. CSLI Publications, Stanford, 1996.

7. J. van Benthem. Dynamic bits and pieces. Technical Report LP-97-01, Institute for Logic, Language and Computation, University of Amsterdam, 1997.

8. R. Berger. The undecidability of the domino problem. Memoirs of the American Mathematical Society, 66: 1-72, 1966.

9. P. Blackburn, M. de Rijke, and Y. Venema. Modal Logic. Cambridge University Press, 2001.

10. E. Börger, E. Grädel, and Y. Gurevich. The Classical Decision Problem. Springer, 1997.

11. A. Borgida. Description logics in data management. IEEE Transactions on Knowledge and Data Engineering, 7:671-682, 1995.

12. J.P. Burgess and Y. Gurevich. The decision problem for linear temporal logic. Notre Dame Journal of Formal Logic, 26:115-128, 1985.

13. D. Calvanese, G. De Giacomo, D. Nardi, and M. Lenzerini. Reasoning in expressive description logics. In A. Robinson and A. Voronkov, editors, Handbook of Automated Reasoning, pages 1581-1634. Elsevier Science, 1999.

14. A. Chagrov and M. Zakharyaschev. Modal Logic. Oxford Logic Guides No. 35. Oxford University Press, Oxford, 1997.

15. A. Chandra and P. Merlin. Optimal implementation of conjunctive queries in relational databases. In Proceedings of 9th ACM Symposium on Theory of Computing, pages 77-90, 1977.

16. M.A.E. Dummett and E.J. Lemmon. Modal logics between S4 and S5. Zeitschrift für mathematische Logik und Grundlagen der Mathematik, 5:250-264, 1959.

17. R. Fagin, J.Y. Halpern, Y. Moses, and M.Y. Vardi. Reasoning about Knowledge. MIT Press, 1995.

18. M. Fischer and R. Ladner. Propositional dynamic logic of regular programs. Journal of Computer and System Sciences, 18(2):194-211, 1979.

19. M. Fürer. The computational complexity of the unconstrained limited domino problem (with implications for logical decision problems). In E. Börger, G. Hasenjaeger and D. Rödding, Logic and Machines: Decision Problems and Complexity. Lecture Notes in Computer Science No. 171, pages 312-319. Springer, 1981.

20. D.M. Gabbay. An irreflexivity lemma with applications to axiomatizations of conditions on linear frames. In U. Mönnich, editor, Aspects of Philosophical Logic, pages 67-89. Reidel, 1981. 
21. E. Grädel. On the restraining power of guards. Journal of Symbolic Logic, 64(4):1719-1742, 1999.

22. E. Grädel and I. Walukiewicz. Guarded fixed point logic. In Proceedings of 14 th IEEE Symposium on Logic in Computer Science LICS '99, Trento, 1999.

23. J.Y. Halpern and Y.O. Moses. A guide to completeness and complexity for modal logics of knowledge and belief. Artificial Intelligence, 54:319-379, 1992.

24. D. Harel. Dynamic logic. In D.M. Gabbay and F. Guenther, editors, Handbook of Philosophical Logic, volume 2, pages 497-604. Reidel, Dordrecht, 1984.

25. R. Harrop. On the existence of finite models and decision procedures for propositional calculi. Proceedings of the Cambridge Philosophical Society, 54:1-13, 1958.

26. L. Henkin. Logical Systems Containing Only a Finite Number of Symbols. Séminiare de Mathematique Supérieures 21. Les Presses de l'Université de Montréal, Montréal, 1967.

27. L. Henkin, J. D. Monk, and A. Tarski. Cylindric Algebras, Parts I and II. North-Holland, 1971 and 1985.

28. M. Hennessy and R. Milner. Algebraic laws for indeterminism and concurrency. Journal of the ACM, 32:137-162, 1985.

29. B. Herwig. Extending partial isomorphisms on finite structures. Combinatorica, 15:365-371, 1995.

30. R. Hirsch, I. Hodkinson, M. Marx, Sz. Mikulás, and M. Reynolds. Mosaics and step-by-step. Remarks on "A modal logic of relations". In E. Orłowska, editor, Logic at Work. Essays Dedicated to the Memory of Elena Rasiowa, Studies in Fuzziness and Soft Computing, pages 158-167. Springer, 1999.

31. W. Hodges. Model Theory. Cambridge University Press, 1993.

32. I. Hodkinson. Loosely guarded fragment of first-order logic has the finite model property. Studia Logica, 70(2):205-240, 2002.

33. N. Immerman. Upper and lower bounds for first-order expressibility. Journal of Computer and System Sciences, 25:76-98, 1982.

34. N. Immerman and D. Kozen. Definability with bounded number of bound variables. In Proceedings of the Symposium on Logic in Computer Science, pages 236-244, Washington, 1987. Computer Society Press.

35. D. Janin and I. Walukiewicz. On the expressive completeness of the propositional $\mu$-calculus w.r.t. monadic second-order logic. In Proceedings of CONCUR '96, 1996.

36. N. Kurtonina and M. de Rijke. Bisimulations for temporal logic. Journal of Logic, Language and Information, 6:403-425, 1997.

37. R. Ladner. The computational complexity of provability in systems of modal propositional logic. SIAM Journal of computing, 6(3):467-480, 1977.

38. E.J. Lemmon. Algebraic semantics for modal logics [Parts I and II]. Journal of Symbolic Logic, pages 46-65 and 191-218, 1966.

39. Z. Manna and A. Pnueli. The Temporal Logic of Reactive and Concurrent Systems. Vol. 1, Specification. Springer, 1992.

40. M. Marx. Complexity of products of modal logics. Journal of Logic and Computation, 9(2):221-238, 1999.

41. M. Marx. Tolerance logic. Journal of Logic, Language and Information 10:353373, 2001.

42. M. Marx, S. Schlobach, and Sz. Mikulás. Labelled deduction for the guarded fragment. In D. Basin et al., editors, Labelled Deduction, Applied Logic Series, pages 193-214. Kluwer Academic, 2000. 
43. M. Marx and Y. Venema. Multi-dimensional Modal Logic. Applied Logic Series. Kluwer Academic, 1997.

44. J.C.C. McKinsey and A. Tarski. The algebra of topology. Annals of Mathematics, pages 141-191, 1944.

45. I. Németi. Free Algebras and Decidability in Algebraic Logic. DSc. thesis, Mathematical Institute of the Hungararian Academy of Sciences, Budapest, 1986 (in Hungarian; English version in [46]).

46. I. Németi. Decidability of weakened versions of first-order logic. In Logic Colloquium '92, pages 177-242, Stanford, 1995. CSLI Publications.

47. M. Otto. Bounded Variable Logics and Counting. A Study in Finite Models. Lecture Notes in Logic No. 9. Springer, 1997.

48. D. Park. Concurrency and automata on infinite sequences. In P. Deussen, editor, Theoretical Computer Science. Lecture Notes in Computer Science No. 104, pages 167-183. Springer, 1981.

49. S. Popkorn. First Steps in Modal Logic. Cambridge University Press, Cambridge, 1992.

50. V. Pratt. Models of program logics. In Proceedings of the 20th IEEE symposium on Foundations of Computer Science, pages 115-122, 1979.

51. E. Rosen. Modal logic over finite structures. Journal of Logic, Language and Information, 6:427-439, 1997.

52. K. Segerberg. An Essay in Classical Modal Logic. Filosofiska Studier 13. University of Uppsala, 1971.

53. K. Segerberg. Two-dimensional modal logic. Journal of Philosophical Logic, 2:77-96, 1973.

54. A. Sistla and E. Clarke. Complexity of propositional linear temporal logics. Journal of the ACM, 32(3):733-749, 1985.

55. E. Spaan. Complexity of Modal Logics. PhD thesis, Institute for Logic, Language and Computation, University of Amsterdam, 1993.

56. C. Stirling. Modal and temporal logics. In S. Abramsky, D.M. Gabbay, and T.S.E. Maibaum, editors, Handbook of Logic in Computer Science I, pages 641761. Clarendon Press, 1992.

57. M. Vardi. On the complexity of bounded-variable queries. In N. Immerman and Ph. G. Kolaitis, editors, Descriptive Complexity and Finite Models, volume 31 of DIMACS Series in Discrete Mathematics and Theoretical Computer Science, pages 149-184. American Mathematical Society, 1996.

58. M. Vardi. Why is modal logic so robustly decidable? In DIMACS Series in Discrete Mathematics and Theoretical Computer Science 31, pages 149-184. American Math. Society, 1997.

59. Y. Venema. Many-Dimensional Modal Logic. PhD thesis, Institute for Logic, Language and Computation, University of Amsterdam, 1992.

60. Y. Venema. Cylindric modal logic. Journal of Symbolic Logic, 60(2):591-623, 1995.

61. H. Wang. Proving theorems by pattern recognition II. Bell Systems Techical Journal, 40:1-41, 1961. 